\begin{tabular}{|c|l|}
\hline Title & $\begin{array}{l}\text { A Family of Inequival ent Weyl Representations of Canonical Commutation Relations with A pplications to Quantum } \\
\text { Field Theory }\end{array}$ \\
\hline Author(s) & A rai, A sao \\
\hline Citation & Hokkaido University Preprint Series in Mathematics, 1085, 1-24 \\
\hline Issue Date & 2015-12-18 \\
\hline DOI & 10.14943/84229 \\
\hline Doc URL & http://hdl.handle.net/2115/69889 \\
\hline Type & bulletin (article) \\
\hline File Information & pre1085.pdf \\
\hline
\end{tabular}

Instructions for use 


\title{
A Family of Inequivalent Weyl Representations of Canonical Commutation Relations with Applications to Quantum Field Theory
}

\author{
Asao Arai \\ Department of Mathematics, Hokkaido University \\ Sapporo, Hokkaido 060-0810 \\ Japan \\ E-mail: arai@math.sci.hokudai.ac.jp
}

\begin{abstract}
Considered is a family of irreducible Weyl representations of canonical commutation relations with infinite degrees of freedom on the abstract boson Fock space over a complex Hilbert space. Theorems on equivalence or inequivalence of the representations are established. As a simple application of one of these theorems, the well known inequivalence of the time-zero field and conjugate momentum for different masses in a quantum scalar field theory is rederived with space dimension $d \geq 1$ arbitrary. Also a generalization of representations of the time-zero field and conjugate momentum is presented. Comparison is made with a quantum scalar field in a bounded region in $\mathbb{R}^{d}$. It is shown that, in the case of a bounded space region with $d=1,2,3$, the representations for different masses turn out to be mutually equivalent.
\end{abstract}

Keywords: Boson Fock space, canonical commutation relations, inequivalent representation, quantum field, time-zero field, Weyl representation.

Mathematics Subject Classification 2010: 81R10, 47L60.

\section{Introduction}

As is well known, in the Fock space formalism of quantum field theory (see, e.g., [6, $\S \mathrm{X} .7])$, the time-zero field $\phi_{m}(f)$ and conjugate momentum $\pi_{m}(f)$ of a free scalar field on the four-dimensional space-time $\mathbb{R}^{3} \times \mathbb{R}$ with mass $m \geq 0$ and $f \in \mathcal{S}_{\mathbb{R}}\left(\mathbb{R}^{3}\right.$ ) (the Schwartz space of rapidly decreasing real-valued $C^{\infty}$-functions on $\mathbb{R}^{3}$ ) give an irreducible Weyl representation of the canonical commutations relations (CCR) over $\mathcal{S}_{\mathbb{R}}\left(\mathbb{R}^{3}\right.$ ) (see Definition 2.4-(ii) and Example 2.6). Namely $\phi_{m}(f)$ and $\pi_{m}(f)$ are self-adjoint operators on the boson Fock space $\mathcal{F}_{\mathrm{b}}\left(L^{2}\left(\mathbb{R}^{3}\right)\right.$ ) over $L^{2}\left(\mathbb{R}^{3}\right)$ (see Section 2 for the definition of the 
abstract boson Fock space over a complex Hilbert space) satisfying the Weyl relations (e.g., [6, Appendix to X.7]):

$$
\begin{aligned}
& e^{i \phi_{m}(f)} e^{i \pi_{m}(g)}=e^{-i\langle f, g\rangle} e^{i \pi_{m}(g)} e^{i \phi_{m}(f)}, \\
& e^{i \phi_{m}(f)} e^{i \phi_{m}(g)}=e^{i \phi_{m}(g)} e^{i \phi_{m}(f)}, \quad e^{i \pi_{m}(f)} e^{i \pi_{m}(g)}=e^{i \pi_{m}(g)} e^{i \pi_{m}(f)}, \quad f, g \in \mathcal{S}_{\mathbb{R}}\left(\mathbb{R}^{3}\right),
\end{aligned}
$$

where $i$ is the imaginary unit and $\langle f, g\rangle$ denotes the inner product of $f$ and $g$ in $L^{2}\left(\mathbb{R}^{3}\right)$. The set $\left\{\phi_{m}(f), \pi_{m}(f) \mid f \in \mathcal{S}_{\mathbb{R}}\left(\mathbb{R}^{3}\right)\right\}$ is also a Heisenberg representaion of the CCR over $\mathcal{S}_{\mathbb{R}}\left(\mathbb{R}^{3}\right)$ (see Definition 2.4-(i) as well as Remark 2.5) :

$$
\begin{aligned}
& {\left[\phi_{m}(f), \pi_{m}(g)\right]=i\langle f, g\rangle,} \\
& {\left[\phi_{m}(f), \phi_{m}(g)\right]=0,\left[\pi_{m}(f), \pi_{m}(g]=0, \quad f, g \in \mathcal{S}_{\mathbb{R}}\left(\mathbb{R}^{3}\right)\right.}
\end{aligned}
$$

on a dense subspace in $\mathcal{F}_{\mathrm{b}}\left(L^{2}\left(\mathbb{R}^{3}\right)\right)$, where $[A, B]:=A B-B A$, the commutator of $A$ and $B$. As for this representation, the following interesting fact is known:

Theorem $1.1\left[6\right.$, Theorem X.46] If $m_{1} \neq m_{2}\left(m_{1}, m_{2}>0\right)$, then the Weyl representations $\left\{e^{i \phi_{m_{1}}(f)}, e^{i \pi_{m_{1}}(f)} \mid f \in \mathcal{S}_{\mathbb{R}}\left(\mathbb{R}^{3}\right)\right\}$ and $\left\{e^{i \phi_{m_{2}}(f)}, e^{i \pi_{m_{2}}(f)} \mid f \in \mathcal{S}_{\mathbb{R}}\left(\mathbb{R}^{3}\right)\right\}$ are inequivalent, i.e., there exists no unitary operator $U$ on $\mathcal{F}_{\mathrm{b}}\left(L^{2}\left(\mathbb{R}^{3}\right)\right)$ such that, for all $f \in \mathcal{S}_{\mathbb{R}}\left(\mathbb{R}^{3}\right)$, $U \phi_{m_{1}}(f) U^{-1}=\phi_{m_{2}}(f)$ and $U \pi_{m_{1}}(f) U^{-1}=\pi_{m_{2}}(f)$.

The proof of this theorem in [6] uses the Euclidean invariance of the operators $\phi_{m}(f)$ and $\pi_{m}(f)$. This comes from "the idea that Euclidean invariance is deeply connected with questions of inequivalence of representations of the CCR" [6, p.329]. But we want to point out that there exists a general abstract structure which, in a concrete realization, makes the representations $\left\{\phi_{m}(f), \pi_{m}(f) \mid f \in \mathcal{S}_{\mathbb{R}}\left(\mathbb{R}^{3}\right)\right\}, m>0$ mutually inequivalent. One of the motivations for the present work is to show this by establishing an abstract theorem on inequivalence of representations of CCR on the abstract boson Fock space (Theorem 5.1) and deriving Theorem 1.1 as an application of the abstract theorem. We see that, from this view-point, the translation invariance in space is connected with the nonHilbert-Schmidtness of an operator which is a necessary and sufficient condition for the representations $\left\{\phi_{m_{1}}(f), \pi_{m_{1}}(f) \mid f \in \mathcal{S}_{\mathbb{R}}\left(\mathbb{R}^{3}\right)\right\}$ and $\left\{\phi_{m_{2}}(f), \pi_{m_{2}}(f) \mid f \in \mathcal{S}_{\mathbb{R}}\left(\mathbb{R}^{3}\right)\right\}\left(m_{1} \neq\right.$ $m_{2}$ ) to be inequivalent. Schematically speaking, the translation invariance in space implies the continuity of the energy spectrum of one free boson, which, in turn, implies the nonHilbert-Schmidtness of the relevant operator.

We also present a generalization of the representation $\left\{\phi_{m}(f), \pi_{m}(f) \mid f \in \mathcal{S}_{\mathbb{R}}\left(\mathbb{R}^{3}\right)\right\}$ in such a way that the energy function $\omega_{m}$ of a free relativistic boson with mass $m$ is replaced by a general function and the space $\mathbb{R}^{3}$ is replaced by $\mathbb{R}^{d}$ with $d \in \mathbb{N}$ (the set of natural numbers) arbitrary. We prove a theorem on equivalence of the representations in the generalized family (Theorem 6.10). Since infinity in space may give rise to inequivalence of representations $\left\{\phi_{m}(f), \pi_{m}(f) \mid f \in \mathcal{S}_{\mathbb{R}}\left(\mathbb{R}^{3}\right)\right\}$, we also discuss a quantum field in a bounded space region in $\mathbb{R}^{d}$. In this case, we find that, in the case $d=1,2,3$, representations of time-zero fields for different masses are mutually equivalent, in contrast to the case of the infinite space $\mathbb{R}^{d}$. This may be an interesting phenomenon to note.

The present paper is organized as follows. In Section 2, we recall the definition of the boson Fock space $\mathcal{F}_{\mathrm{b}}(\mathcal{H})$ over a complex Hilbert space $\mathcal{H}$ and describe some facts. Also 
concepts of representations of CCR over a real inner product space are reviewed. Some details of the above mentioned representation of CCR are given as an example, where the space dimension $d$ is taken to be arbitrary (not necessarily $d=3$ ). In Section 3, we describe the Fock representation of CCR over a real subspace $\mathcal{V}$ in $\mathcal{H}$. We prove a key fact in considering inequivalence of representations of CCR (Proposition 3.3). In Section 4, we introduce a family $\mathcal{W}$ of irreducible Weyl representation of CCR over a real subspace $\mathcal{V}$ in $\mathcal{H}$. This family is parameterized by a set $S_{\mathcal{V}}(\mathcal{H})$ consisting of injective self-adjoint operators with some additional properties. We prove a theorem on inequivalence of a representation in $\mathcal{W}$ to the Fock representation (Theorem 4.6). In Section 5, we state and prove the main theorems in the present paper (Theorems 5.1 and 5.6), which are concerned with equivalence or inequivalence of two representations in $\mathcal{W}$. A basic idea for proof of Theorem 5.1 is to reduce the problem to that of Bogoliubov transformations on the creation and annihilation operators on $\mathcal{F}_{\mathrm{b}}(\mathcal{H})$. As for Theorem 5.6, we apply Proposition 3.3 mentioned above. In the last section, we first show that Theorem 1.1 can be obtained by an application of Theorem 5.1. Moreover, as mentioned above, we define a generalized version of the representations $\left\{\phi_{m}(f), \pi_{m}(f) \mid f \in \mathcal{S}_{\mathbb{R}}\left(\mathbb{R}^{3}\right)\right\}$ and prove a theorem on inequivalence of them (Theorem 6.10). Finally, for comparison, we discuss a scalar quantum field with mass $m$ in a bounded region $M \subset \mathbb{R}^{d}$. We show that, in the case $d=1,2,3$, representations for different masses are equivalent, opposite to the case where the space under consideration is $\mathbb{R}^{d}$.

\section{Preliminaries}

\subsection{The abstract boson Fock space and basic facts}

Let $\mathcal{H}$ be a complex Hilbert space with inner product $\langle\cdot, \cdot\rangle$ (linear in the second variable) and norm $\|\cdot\|$, and, for each non-negative integer $n=0,1,2, \ldots, \otimes_{\mathrm{s}}^{n} \mathcal{H}$ be the $n$-fold symmetric tensor product Hilbert space of $\mathcal{H}$ with convention $\otimes_{\mathrm{s}}^{0} \mathcal{H}:=\mathbb{C}$ (the complex number field). Then the boson Fock space over $\mathcal{H}$ is defined by

$$
\mathcal{F}_{\mathrm{b}}(\mathcal{H}):=\oplus_{n=0}^{\infty} \otimes_{\mathrm{s}}^{n} \mathcal{H}
$$

For a linear operator $A$ on a Hilbert space, we denote its domain by $D(A)$.

We denote by $A(f)$ the annihilation operator with test vector $f \in \mathcal{H}$ on $\mathcal{F}_{\mathrm{b}}(\mathcal{H})$, which is the unique densely defined closed operator on $\mathcal{F}_{\mathrm{b}}(\mathcal{H})$ such that its adjoint $A(f)^{*}$ takes the following form: For all $\Psi \in D\left(A(f)^{*}\right),\left(A(f)^{*} \Psi\right)^{(0)}=0$ and

$$
\left(A(f)^{*} \Psi\right)^{(n)}=\sqrt{n} S_{n}\left(f \otimes \Psi^{(n-1)}\right), \quad n \geq 1,
$$

where $S_{n}$ is the symmetrization operator on the $n$-fold tensor product $\otimes^{n} \mathcal{H}$ of $\mathcal{H}$ (see, e.g., $[5, \S I I .4]$ and $[6, \S$ X.7]). It is well known that the subspace of finite particle vectors

$$
\begin{aligned}
\mathcal{F}_{0}(\mathcal{H}):=\left\{\Psi=\left\{\Psi^{(n)}\right\}_{n=0}^{\infty} \quad\right. & \Psi^{(n)} \in \otimes_{\mathrm{s}}^{n} \mathcal{H}, n \geq 0 \text { and there exists an } n_{0} \in \mathbb{N} \text { such that } \\
& \text { for all } \left.n \geq n_{0}, \Psi^{(n)}=0\right\}
\end{aligned}
$$

is dense in $\mathcal{F}_{\mathrm{b}}(\mathcal{H})$. 
For all $f \in \mathcal{H}, \mathcal{F}_{0}(\mathcal{H}) \subset D(A(f)) \cap D\left(A(f)^{*}\right)$ and $A(f)$ and $A(f)^{*}$ leave $\mathcal{F}_{0}(\mathcal{H})$ invariant. Moreover, $\left\{A(f), A(f)^{*} \mid f \in \mathcal{H}\right\}$ satisfies the following commutation relations:

$$
\left[A(f), A(g)^{*}\right]=\langle f, g\rangle, \quad[A(f), A(g)]=0, \quad\left[A(f)^{*}, A(g)^{*}\right]=0 \quad(f, g \in \mathcal{H})
$$

on $\mathcal{F}_{0}(\mathcal{H})$.

We denote by $A(f)^{\#}$ either $A(f)$ or $A(f)^{*}$.

Let

$$
\Omega_{\mathrm{F}}=\{1,0,0, \ldots\} \in \mathcal{F}_{\mathrm{b}}(\mathcal{H})
$$

be the Fock vacuum. Then

$$
A(f) \Omega_{\mathrm{F}}=0, \quad f \in \mathcal{H} .
$$

Let $N_{\mathrm{b}}$ be the number operator on $\mathcal{F}_{\mathrm{b}}(\mathcal{H})$ :

$$
N_{\mathrm{b}}=\oplus_{n=0}^{\infty} n I_{n}
$$

where $I_{n}$ is the identity on $\otimes_{\mathrm{s}}^{n} \mathcal{H}$.

It is well known (e.g., [1, Proposition 4.25], [2, Theorem 3.51]) that, for all $f \in \mathcal{H}$, $D\left(N_{\mathrm{b}}^{1 / 2}\right) \subset D(A(f)) \cap D\left(A(f)^{*}\right)$ and

$$
\|A(f) \Psi\| \leq\|f\|\left\|N_{\mathrm{b}}^{1 / 2} \Psi\right\|, \quad\left\|A(f)^{*} \Psi\right\| \leq\|f\|\left\|\left(N_{\mathrm{b}}+1\right)^{1 / 2} \Psi\right\|, \quad \Psi \in D\left(N_{\mathrm{b}}^{1 / 2}\right) .
$$

Hence we have the following fact:

Lemma 2.1 (strong continuity of $A(\cdot)^{\#}$ on $D\left(N_{\mathrm{b}}^{1 / 2}\right)$ ) Let $\Psi \in D\left(N_{\mathrm{b}}^{1 / 2}\right)$ and $f_{n}, f \in \mathcal{H}$ $(n \in \mathbb{N})$ satisfying $\lim _{n \rightarrow \infty} f_{n}=f$. Then $\lim _{n \rightarrow \infty} A\left(f_{n}\right)^{\#} \Psi=A(f)^{\#} \Psi$.

For each $f \in \mathcal{H},\left(A(f)^{*}+A(f)\right) / \sqrt{2}$ is essentially self-adjoint on $\mathcal{F}_{0}(\mathcal{H})[6$, Theorem X.41]. Hence its closure

$$
\Phi(f):=\frac{1}{\sqrt{2}} \overline{\left(A(f)^{*}+A(f)\right)} \quad(f \in \mathcal{H})
$$

is self-adjoint, where, for a closable operator $T, \bar{T}$ denotes the closure of $T$. The operator $\Phi(f)$ is called the Segal field operator.

It follows from (2.3) that, for all $f, g \in \mathcal{H}$,

$$
[\Phi(f), \Phi(g)]=i \Im\langle f, g\rangle
$$

on $\mathcal{F}_{0}(\mathcal{H})$. Moreover, $\{\Phi(f) \mid f \in \mathcal{H}\}$ obeys the following relations:

$$
e^{i \Phi(f)} e^{i \Phi(g)}=e^{-i \Im\langle f, g\rangle} e^{i \Phi(g)} e^{i \Phi(f)}, \quad f, g \in \mathcal{H} .
$$

For each $f \in \mathcal{H}$, the operator

$$
\Pi(f):=\Phi(i f)
$$

is called the conjugate momentum of $\Phi(f)$. We have from (2.8)

$$
[\Phi(f), \Pi(g)]=i \Re\langle f, g\rangle, \quad[\Pi(f), \Pi(g)]=i \Im\langle f, g\rangle, \quad f, g \in \mathcal{H} .
$$


Lemma 2.2 Let $\mathcal{D}$ be a dense subspace in $\mathcal{H}$ and $\Psi \in \mathcal{F}_{\mathrm{b}}(\mathcal{H})$ such that, for all $f \in \mathcal{D}$, $\Psi \in D(A(f))$ and

$$
A(f) \Psi=0, \quad f \in \mathcal{D} .
$$

Then $\Psi=\Psi^{(0)} \Omega_{\mathrm{F}}$, where $\Omega_{\mathrm{F}}$ is the Fock vacuum.

Proof. Equation (2.12) implies that $(A(f) \Psi)^{(n)}=0, n \geq 0$. Hence, for all $\Phi_{n} \in \otimes_{\mathrm{s}}^{n} \mathcal{H}$, $\left\langle\Phi_{n}, A(f) \Psi\right\rangle=0$ (we identify $\Phi_{n}$ with $\left\{0, \ldots, \Phi_{n}, 0, \ldots\right\} \in \mathcal{F}_{0}(\mathcal{H})$ ). Since $\Phi_{n} \in D\left(A(f)^{*}\right)$, it follows that $\left\langle A(f)^{*} \Phi_{n}, \Psi\right\rangle=0$. Since $\left\{A(f)^{*} \Phi_{n} \mid f \in \mathcal{D}, \Phi_{n} \in \otimes_{\mathrm{s}}^{n} \mathcal{H}\right\}$ is dense in $\otimes_{\mathrm{s}}^{n+1} \mathcal{H}$, we have $\Psi^{(n+1)}=0$ for all $n \geq 0$. Hence $\Psi=\left\{\Psi^{(0)}, 0, \ldots\right\}=\Psi^{(0)} \Omega_{\mathrm{F}}$.

Lemma 2.3 Assume that $\mathcal{H}$ is separable and $\left\{e_{n}\right\}_{n=1}^{\infty}$ be a complete orthonormal system $(C O N S)$ of $\mathcal{H}$. Then, for all $\Psi \in D\left(N_{\mathrm{b}}^{1 / 2}\right), \sum_{n=1}^{\infty}\left\|A\left(e_{n}\right) \Psi\right\|^{2}$ converges and

$$
\sum_{n=1}^{\infty}\left\|A\left(e_{n}\right) \Psi\right\|^{2}=\left\|N_{\mathrm{b}}^{1 / 2} \Psi\right\|^{2}
$$

Proof. This fact may be well known, but, for completeness, we give a proof for it. Since $\mathcal{H}$ is separable, there exists a unitary operator $U$ from $\mathcal{H}$ to $L^{2}(\mathbb{R})$. Then $\Gamma(U):=$ $1 \oplus\left(\oplus_{n=1}^{\infty} \otimes^{n} U\right)$ is a unitary operator from $\mathcal{F}_{\mathrm{b}}(\mathcal{H})$ to $\mathcal{F}_{\mathrm{b}}\left(L^{2}(\mathbb{R})\right)$ such that

$$
\begin{aligned}
& \Gamma(U) A(f) \Gamma(U)^{-1}=a(U f), \quad f \in \mathcal{H}, \\
& \Gamma(U) N_{\mathrm{b}} \Gamma(U)^{-1}=n_{\mathrm{b}},
\end{aligned}
$$

where $a(\cdot)$ (resp. $n_{\mathrm{b}}$ ) denotes the annihilation (resp. number) operator on $\mathcal{F}_{\mathrm{b}}\left(L^{2}(\mathbb{R})\right)$. Hence (2.13) is equivalent to

$$
\sum_{n=1}^{\infty}\left\|a\left(f_{n}\right) \psi\right\|^{2}=\left\|n_{\mathrm{b}}^{1 / 2} \psi\right\|^{2}, \quad \psi \in D\left(n_{\mathrm{b}}^{1 / 2}\right),
$$

where $f_{n}=U e_{n}, n \in \mathbb{N}$. We prove this formula. It is well known or easy to see (e.g., [6, p.209, Example 1]) that, for all $\psi \in D(a(f))$ with $f \in L^{2}(\mathbb{R})$ and $\ell \geq 0$

$$
(a(f) \psi)^{(\ell)}\left(x_{1}, \ldots, x_{\ell}\right)=\sqrt{\ell+1} \int_{\mathbb{R}} f(x)^{*} \psi^{(\ell+1)}\left(x, x_{1}, \ldots, x_{\ell}\right) d x
$$

for a.e. $\left(x_{1}, \ldots, x_{\ell}\right) \in \mathbb{R}^{\ell}$, where, for $z \in \mathbb{C}, z^{*}$ denotes the complex conjugate of $z$. Hence, for all $\psi \in D\left(n_{\mathrm{b}}^{1 / 2}\right)$,

$$
\left\|a\left(f_{n}\right) \psi\right\|^{2}=\sum_{\ell=0}^{\infty}(\ell+1) \int_{\mathbb{R}^{\ell}}\left|\int_{\mathbb{R}} f_{n}(x)^{*} \psi^{(\ell+1)}\left(x, x_{1}, \ldots, x_{\ell}\right) d x\right|^{2} d x_{1} \cdots d x_{\ell} .
$$

By the completeness of $\left\{f_{n}\right\}_{n}$, we have

$$
\sum_{n=1}^{\infty}\left|\int_{\mathbb{R}} f_{n}(x)^{*} \psi^{(\ell+1)}\left(x, x_{1}, \ldots, x_{\ell}\right) d x\right|^{2}=\int_{\mathbb{R}}\left|\psi^{(\ell+1)}\left(x, x_{1}, \ldots, x_{\ell}\right)\right|^{2} d x .
$$

Hence, by Fubini's theorem,

$$
\sum_{n=1}^{\infty}\left\|a\left(f_{n}\right) \psi\right\|^{2}=\sum_{\ell=0}^{\infty}(\ell+1)\left\|\psi^{(\ell+1)}\right\|^{2}=\left\|n_{\mathrm{b}}^{1 / 2} \psi\right\|^{2} .
$$

Thus (2.14) holds. 


\subsection{Representations of CCR over a real inner product space}

We recall concepts of representation of the CCR over a real inner product space.

Definition 2.4 Let $\mathcal{F}$ be a complex Hilbert space, $\mathcal{F}_{0}$ be a dense subspace in $\mathcal{F}$ and $\mathcal{V}$ be a real inner product space. Suppose that, for each $f \in \mathcal{V}$, closed symmetric operators $q(f)$ and $p(f)$ on $\mathcal{F}$ are given.

(i) The triple $\left(\mathcal{F}, \mathcal{F}_{0},\{q(f), p(f) \mid f \in \mathcal{V}\}\right)$ is called a Heisenberg representation of the $C C R$ over $\mathcal{V}$ if, for all $f \in \mathcal{V}, \mathcal{F}_{0} \subset D(q(f)) \cap D(p(f))$ and $q(f)$ and $p(f)$ leave $\mathcal{F}_{0}$ invariant, satisfying the CCR

$$
[q(f), p(g)]=i\langle f, g\rangle, \quad[q(f), q(g)]=0, \quad[p(f), p(g)]=0, \quad f, g \in \mathcal{V},
$$

on $\mathcal{F}_{0}$.

(ii) Assume that, for each $f \in \mathcal{V}, q(f)$ and $p(f)$ are self-adjoint. Then $\left(\mathcal{F},\left\{e^{i q(f)}\right.\right.$, $\left.\left.e^{i p(f)} \mid f \in \mathcal{V}\right\}\right)$ is called a Weyl representation of the $C C R$ over $\mathcal{V}$ if the Weyl relations

$$
\begin{aligned}
& e^{i q(f)} e^{i p(g)}=e^{-i\langle f, g\rangle} e^{i p(g)} e^{i q(f)}, \\
& e^{i q(f)} e^{i q(g)}=e^{i q(g)} e^{i q(f)}, \quad e^{i p(f)} e^{i p(g)}=e^{i p(g)} e^{i p(f)}, \quad f, g \in \mathcal{V},
\end{aligned}
$$

hold.

(iii) Let $\rho:=\left(\mathcal{F}, \mathcal{F}_{0},\{q(f), p(f) \mid f \in \mathcal{V}\}\right)$ and $\rho^{\prime}=\left(\mathcal{F}^{\prime}, \mathcal{F}_{0}^{\prime},\left\{q(f)^{\prime}, p(f)^{\prime} \mid f \in \mathcal{V}\right\}\right)$ be Heisenberg representations of the CCR over $\mathcal{V}$. Then $\rho$ and $\rho^{\prime}$ are equivalent if there exists a unitary operator $U: \mathcal{F} \rightarrow \mathcal{F}^{\prime}$ such that $U q(f) U^{-1}=q(f)^{\prime}, U p(f) U^{-1}=$ $p(f)^{\prime}$ for all $f \in \mathcal{V}$.

(iv) Let $\rho:=\left(\mathcal{F},\left\{e^{i q(f)}, e^{i p(f)} \mid f \in \mathcal{V}\right\}\right)$ and $\rho^{\prime}=\left(\mathcal{F}^{\prime},\left\{e^{i q(f)^{\prime}}, e^{i p(f)^{\prime}} \mid f \in \mathcal{V}\right\}\right)$ be Weyl representations of the CCR over $\mathcal{V}$. Then $\rho$ and $\rho^{\prime}$ are equivalent if there exists a unitary operator $U: \mathcal{F} \rightarrow \mathcal{F}^{\prime}$ such that $U q(f) U^{-1}=q(f)^{\prime}, U p(f) U^{-1}=p(f)^{\prime}$ for all $f \in \mathcal{V}$.

Remark 2.5 (i) In our definition, the operators forming a Heisenberg representation are not necessarily self-adjoint.

(ii) A Weyl representation $\left(\mathcal{F},\left\{e^{i q(f)}, e^{i p(f)} \mid f \in \mathcal{V}\right\}\right)$ is a Heisenberg representation $\left(\mathcal{F}, \mathcal{F}_{0},\{q(f), p(f) \mid f \in \mathcal{V}\}\right)$ for a suitable $\mathcal{F}_{0}$. But the converse is not true. This situation already occurs in the case where $\mathcal{V}$ is finite dimensional (cf., e.g., [4]).

(iii) In the case where $\mathcal{V}$ is finite dimensional, all irreducible Weyl representations of the CCR over $\mathcal{V}$ are mutually equivalent (von Neumann's uniqueness theorem [4]). But, as for Heisenberg representations, von Neumann's uniqueness theorem does not hold in general.

Example 2.6 For each $d \in \mathbb{N}$, there exists a $d$-dimensional version of the time-zero field $\phi_{m}(f)$ and conjugate momentum $\pi_{m}(f)$ mentioned in Introduction. Here we describe it briefly. Let $\Delta$ be the generalized Laplacian acting in $L^{2}\left(\mathbb{R}^{d}\right)$. Then, for a constant $m \geq 0$,

$$
h_{m}:=\left(-\Delta+m^{2}\right)^{1 / 2}
$$


is a non-negative self-adjoint operator on $L^{2}\left(\mathbb{R}^{d}\right)$, denoting physically the Hamiltonian of a free relativistic particle with mass $m$ in the coordinate representation, where we use the physical unit system such that $\hbar$ (the Planck constant divided by $2 \pi$ ) and $c$ (the speed of light) are 1.

We denote by $\mathbb{R}^{d *}:=\left\{k=\left(k_{1}, \ldots, k_{d}\right) \mid k_{j} \in \mathbb{R}, j=1, \ldots, d\right\}$ the dual space of $\mathbb{R}^{d}$ and by $k x:=\sum_{j=1}^{d} k_{j} x_{j}\left(k \in \mathbb{R}^{d *}, x=\left(x_{1}, \ldots, x_{d}\right) \in \mathbb{R}^{d}\right)$ the natural bilinear form: $\mathbb{R}^{d *} \times \mathbb{R}^{d} \rightarrow \mathbb{R}$

The function $\omega_{m}$ on $\mathbb{R}^{d *}$ given by

$$
\omega_{m}(k):=\sqrt{|k|^{2}+m^{2}}, \quad k \in \mathbb{R}^{d *},
$$

represents the energy function of a free relativistic particle with mass $m$.

Let $\mathcal{F}_{d}: L^{2}\left(\mathbb{R}^{d}\right) \rightarrow L^{2}\left(\mathbb{R}^{d *}\right)$ be the Fourier transform:

$$
\left(\mathcal{F}_{d} f\right)(k)=\hat{f}(k):=\frac{1}{(2 \pi)^{d / 2}} \int_{\mathbb{R}^{d}} f(x) e^{-i k x} d x, \quad\left(k \in \mathbb{R}^{d *}\right)
$$

in the $L^{2}$-sense. Then

$$
D\left(h_{m}\right)=\left\{f \in L^{2}\left(\mathbb{R}^{d}\right) \mid \omega_{m} \hat{f} \in L^{2}\left(\mathbb{R}^{d *}\right)\right\}
$$

and

$$
\widehat{h_{m} f}(k)=\omega_{m}(k) \hat{f}(k), \quad f \in D\left(h_{m}\right), \text { a.e. } k \in \mathbb{R}^{d *} .
$$

We denote by $\mathcal{S}\left(\mathbb{R}^{d}\right)$ (resp. $\mathcal{S}_{\mathbb{R}}\left(\mathbb{R}^{d}\right)$ ) the Schwartz space of (resp. real-valued) rapidly decreasing $C^{\infty}$-functions on $\mathbb{R}^{d}$ and by $L_{\mathbb{R}}^{2}\left(\mathbb{R}^{d}\right)$ the real Hilbert space of real-valued $L^{2}$ functions on $\mathbb{R}^{d}$. We introduce a dense subspace in $\mathcal{V}_{d, m}$ of $L_{\mathbb{R}}\left(\mathbb{R}^{d}\right)$ :

$$
\mathcal{V}_{d, m}:=\left\{\begin{array}{cc}
\mathcal{S}_{\mathbb{R}}\left(\mathbb{R}^{d}\right) & \text { for } m>0, \\
\left\{f \in \mathcal{S}_{\mathbb{R}}\left(\mathbb{R}^{d}\right) \mid \operatorname{supp} \hat{f} \subset \mathbb{R}^{d *} \backslash\{0\}\right\} & \text { for } m=0,
\end{array},\right.
$$

where supp $\hat{f}$ denotes the support of $\hat{f}$. It follows that, for all $\alpha \in \mathbb{R}, \mathcal{V}_{d, m} \subset D\left(h_{m}^{\alpha}\right)$.

We now consider the Fock space $\mathcal{F}_{\mathrm{b}}\left(L^{2}\left(\mathbb{R}^{d}\right)\right)$ over $L^{2}\left(\mathbb{R}^{d}\right)$. For each $f \in \mathcal{V}_{d, m}$, we define

$$
\phi_{m}(f):=\Phi\left(h_{m}^{-1 / 2} f\right), \quad \pi_{m}(f):=\Pi\left(h_{m}^{1 / 2} f\right),
$$

where $\Phi(\cdot)$ and $\Pi(\cdot)$ are respectively the Segal field operator and its conjugate momentum on $\mathcal{F}_{\mathrm{b}}\left(L^{2}\left(\mathbb{R}^{d}\right)\right)$. It is shown that $\left(\mathcal{F}_{\mathrm{b}}\left(L^{2}\left(\mathbb{R}^{d}\right)\right),\left\{e^{i \phi_{m}(f)}, e^{i \pi_{m}(f)} \mid f \in \mathcal{V}_{d, m}\right\}\right)$ is an irreducible Weyl representation of the CCR over $\mathcal{V}_{d, m}$ (see [6, Appendix to X.7]). This representation with $d=3$ is the representation mentioned in Introduction.

\section{Fock representation of CCR and Basic Facts}

Let $\mathcal{H}$ be a complex Hilbert space and $C$ be a conjugation on $\mathcal{H}$ (i.e., $C$ is an anti-linear, norm-preserving map: $\mathcal{H} \rightarrow \mathcal{H}$ satisfying $C^{2}=I$ (identity)). Then the subset

$$
\mathcal{H}_{C}:=\{f \in \mathcal{H} \mid C f=f\}
$$


is a real Hilbert space with the inner product of $\mathcal{H}$. We call $\mathcal{H}_{C}$ the real Hilbert space associated with $C$.

For each $f \in \mathcal{H}$, the vectors

$$
\Re f:=\frac{f+C f}{2}, \quad \Im f:=\frac{f-C f}{2 i}
$$

are elements in $\mathcal{H}_{C}$ satisfying

$$
f=\Re f+i \Im f
$$

We call $\Re f$ (resp. $\Im f$ ) the real (imaginary) part of $f$. We have

$$
\mathcal{H}=\mathcal{H}_{C}+i \mathcal{H}_{C}:=\left\{f+i g \mid f, g \in \mathcal{H}_{C}\right\}
$$

We define

$$
\phi(f):=\Phi(f), \quad \pi(f):=\Pi(f), \quad f \in \mathcal{H}_{C} .
$$

Let $\mathcal{V}$ be a dense subspace in $\mathcal{H}_{C}$. Then $\left(\mathcal{F}_{\mathrm{b}}(\mathcal{H}),\left\{e^{i \phi(f)}, e^{i \pi(f)} \mid f \in \mathcal{V}\right\}\right)$ is an irreducible Weyl representation of the CCR over $\mathcal{V}$ (cf. [6, p.232, Lemma 1]). This representation is called the Fock representation of the CCR over $\mathcal{V}$.

Equation (2.5) gives

$$
(\phi(f)+i \pi(f)) \Omega_{\mathrm{F}}=0, \quad f \in \mathcal{H}_{C} .
$$

By an analogy with this fact, for a Heisenberg representation $\rho_{\mathrm{H}}:=\left(\mathcal{F}, \mathcal{F}_{0},\{q(f), p(f)\right.$ $\mid f \in \mathcal{V}\})$ (resp. a Weyl representation $\rho_{\mathrm{W}}:=\left(\mathcal{F},\left\{e^{i q(f)}, e^{i p(f)} \mid f \in \mathcal{V}\right\}\right)$ ), a non-zero vector $\Omega \in \mathcal{F}$ is called a vacuum of $\rho_{\mathrm{H}}$ (resp. $\left.\rho_{\mathrm{W}}\right)$ if $\Omega \in D(q(f)) \cap D(p(f))$ for all $f \in \mathcal{V}$ and

$$
(q(f)+i p(f)) \Omega=0, \quad f \in \mathcal{V} .
$$

Lemma 3.1 Let $\left(\mathcal{F}, \mathcal{F}_{0},\{q(f), p(f) \mid f \in \mathcal{V}\}\right)$ be a Heisenberg representation of the CCR over $\mathcal{V}$. Suppose that $\left(\mathcal{F}, \mathcal{F}_{0},\{q(f), p(f) \mid f \in \mathcal{V}\}\right)$ is equivalent to the Fock representation $\left(\mathcal{F}_{\mathrm{b}}(\mathcal{H}), \mathcal{F}_{0}(\mathcal{H}),\{\phi(f), \pi(f) \mid f \in \mathcal{V}\}\right)$. Then the representation $\left(\mathcal{F}, \mathcal{F}_{0},\{q(f), p(f) \mid f \in \mathcal{V}\}\right)$ has a vacuum.

Proof. By the present assumption, there exists a unitary operator $U: \mathcal{F} \rightarrow \mathcal{F}_{\mathrm{b}}(\mathcal{H})$ such that

$$
U q(f) U^{-1}=\phi(f), \quad U p(f) U^{-1}=\pi(f), \quad f \in \mathcal{V} .
$$

Hence

$$
U(q(f)+i p(f)) U^{-1}=\phi(f)+i \pi(f) .
$$

Hence it follows from (3.4) that $\Omega:=U^{-1} \Omega_{\mathrm{F}} \in D(q(f)) \cap D(p(f))$ and (3.5) holds. Thus this $\Omega$ is a vacuum of $\left(\mathcal{F}, \mathcal{F}_{0},\{q(f), p(f) \mid f \in \mathcal{V}\}\right)$.

Remark 3.2 The converse of the statement of (3.1) is true under some additional conditions (see, e.g., [1, Proposition 4.61]). 
We denote by $\mathfrak{B}(\mathcal{H})$ the Banach space of everywhere defined bounded linear operators on $\mathcal{H}$.

The following proposition is a key fact in considering inequivalence of representations of CCR.

Proposition 3.3 Assume that $\mathcal{H}$ is separable. Let $L \in \mathfrak{B}(\mathcal{H})$ and $\mathcal{D}$ be a dense subspace in $\mathcal{H}$. Suppose that there exists a non-zero vector $\Omega \in \mathcal{F}_{\mathrm{b}}(\mathcal{H})$ such that, for all $\Psi \in \mathcal{F}_{0}(\mathcal{H})$ and $f \in \mathcal{D}$,

$$
\left\langle A(f)^{*} \Psi, \Omega\right\rangle=\langle A(C L f) \Psi, \Omega\rangle .
$$

Then $L$ is Hilbert-Schmidt.

Proof. For each $n \geq 0$, we define a vector $\Omega_{n}$ in the $n$-particle space $\otimes_{\mathrm{s}}^{n} \mathcal{H}$ by $\Omega_{n}^{(n)}:=$ $\Omega^{(n)}$ and $\Omega_{n}^{(m)}:=0, m \neq n$. Let $\Psi \in \otimes_{\mathrm{s}}^{n} \mathcal{H}$. Then, for all $f \in \mathcal{D}, A(f)^{*} \Psi \in \otimes_{\mathrm{s}}^{n+1} \mathcal{H}$ and $A(C L f) \Psi \in \otimes_{\mathrm{s}}^{n-1} \mathcal{H}\left(\otimes_{\mathrm{s}}^{-1} \mathcal{H}:=\{0\}\right)$. Hence we have from $(3.6)\left\langle A(f)^{*} \Psi, \Omega_{n+1}\right\rangle=$ $\left\langle A(C L f) \Psi, \Omega_{n-1}\right\rangle$. Therefore $\left\langle\Psi, A(f) \Omega_{n+1}\right\rangle=\left\langle\Psi, A(C L f)^{*} \Omega_{n-1}\right\rangle$. Thus

$$
A(f) \Omega_{n+1}=A(C L f)^{*} \Omega_{n-1}, \quad n \geq 0, f \in \mathcal{D},
$$

where $\Omega_{-1}:=0$. In particular, $A(f) \Omega_{1}=0$. Hence, by Lemma $2.2, \Omega_{1}=\left\langle\Omega_{\mathrm{F}}, \Omega_{1}\right\rangle \Omega_{\mathrm{F}}=0$. Then, by (3.7), $A(f) \Omega_{3}=0$ for all $f \in \mathcal{D}$. Hence, by Lemma 2.2 again, $\Omega_{3}=0$. By repeating this process with (3.7), we can show by induction in $n$ that $\Omega_{2 n-1}=0$ for all $n>1$.

By (3.7), we have $A(f) \Omega_{2}=c A(C L f)^{*} \Omega_{\mathrm{F}}$, where $c:=\Omega^{(0)} \in \mathbb{C}$. If $c=0$, then $A(f) \Omega_{2}=0$ for all $f \in \mathcal{D}$. Hence, by Lemma 2.2 again, $\Omega_{2}=0$. Then, in the same way as above, we obtain $\Omega_{2 n}=0$ for all $n \geq 0$. Thus $\Omega=0$. But this is a contradiction. Hence $c \neq 0$. Therefore $\left\|A(C L f)^{*} \Omega_{\mathrm{F}}\right\|^{2}=\left\|A(f) \Omega_{2}\right\|^{2} /|c|^{2}$. Hence $\|L f\|^{2}=\left\|A(f) \Omega_{2}\right\|^{2} /|c|^{2}$. Let $\left\{e_{n}\right\}_{n=1}^{\infty}$ be a CONS of $\mathcal{H}$. Then, by Lemma 2.3,

$$
\sum_{n=1}^{\infty}\left\|L e_{n}\right\|^{2}=\frac{1}{|c|^{2}} \sum_{n=1}^{\infty}\left\|A\left(e_{n}\right) \Omega_{2}\right\|^{2}=\frac{1}{|c|^{2}}\left\|N_{\mathrm{b}}^{1 / 2} \Omega_{2}\right\|^{2}<\infty,
$$

Hence $L$ is Hilbert-Schmidt.

\section{A Family of Irreducible Weyl Representations of CCR}

Let $T$ be a self-adjoint operator on $\mathcal{H}$ (not necessarily bounded) such that

$$
C T \subset T C .
$$

Then $T$ is reduced by $\mathcal{H}_{C}$ in the sense that, for all $f \in D(T), \Re f$ in $\mathcal{H}_{C} \cap D(T)$ and $\Re(T f)=T \Re f$. In particular, $D(T) \cap \mathcal{H}_{C}$ is dense in $\mathcal{H}_{C}$ and $T\left(D(T) \cap \mathcal{H}_{C}\right) \subset \mathcal{H}_{C}$.

Suppose that $T$ is injective. Then it follows from functional calculus that $T^{-1}$ is reduced by $\mathcal{H}_{C}$. We introduce self-adjoint operators

$$
\begin{aligned}
\phi_{T}(f) & :=\Phi\left(T^{-1} f\right), \quad f \in D\left(T^{-1}\right) \cap \mathcal{H}_{C}, \\
\pi_{T}(f) & :=\Pi(T f), \quad f \in D(T) \cap \mathcal{H}_{C},
\end{aligned}
$$


acting in $\mathcal{F}_{\mathrm{b}}(\mathcal{H})$.

Let $\mathcal{V}$ be a dense subspace in $\mathcal{H}_{C}$ and $S_{\mathcal{V}}(\mathcal{H})$ be the set of injective self-adjoint operators $T$ on $\mathcal{H}$ satisfying the following conditions:

(T.1) (4.1) holds.

$(\mathrm{T} .2) \mathcal{V} \subset D(T) \cap D\left(T^{-1}\right)$ and $T^{ \pm 1} \mathcal{V}$ are dense in $\mathcal{H}_{C}$.

Remark 4.1 The set $\mathcal{S}_{\mathcal{V}}(\mathcal{H})$ includes the following sets:

$\mathcal{S}_{\mathcal{V}, \mathrm{c}}(\mathcal{H}):=\left\{T \mid T\right.$ is injective, self-adjoint, $C T \subset T C$ and $\mathcal{V}$ is a core for $\left.T^{ \pm 1}\right\}$, $\mathrm{GL}_{\text {s.a. }}\left(\mathcal{H}_{C}\right):=\{T \in \mathfrak{B}(\mathcal{H}) \mid T$ is self-adjoint, biijective and $T C=C T\}$.

In what follows, we assume that $T \in \mathrm{S}_{\mathcal{V}}(\mathcal{H})$.

Lemma 4.2 The triple $\left(\mathcal{F}_{\mathrm{b}}(\mathcal{H}), \mathcal{F}_{0}(\mathcal{H}),\left\{\phi_{T}(f), \pi_{T}(f) \mid f \in \mathcal{V}\right\}\right)$ is a Heisenberg representation of the CCR over $\mathcal{V}$.

Proof. For all $f, g \in \mathcal{V}$, the CCR for $\phi_{T}(f)$ and $\pi_{T}(g)$ on $\mathcal{F}_{0}(\mathcal{H})$ follow from $(2.11)$ and the elementary fact that $\left\langle T^{-1} f, T g\right\rangle=\langle f, g\rangle$.

Lemma 4.3 Assume that $\mathcal{H}$ is separable. Then $\left(\mathcal{F}_{\mathrm{b}}(\mathcal{H}),\left\{e^{i \phi_{T}(f)}, e^{i \pi_{T}(f)} \mid f \in \mathcal{V}\right\}\right)$ is an irreducible Weyl representation of the CCR over $\mathcal{V}$.

Proof. The Weyl relations for $e^{i \phi_{T}(\cdot)}$ and $e^{i \pi_{T}(\cdot)}$ follow from (2.9) and the fact that $\left\langle T^{-1} f, T g\right\rangle=\langle f, g\rangle, f, g \in \mathcal{V}$. One can show that $\left\{e^{i \Phi(f)}, e^{i \Pi(f)} \mid f \in \mathcal{H}_{C}\right\}$ is irreducible (cf. [6, p.232, Lemma 1]). Let $B \in \mathfrak{B}\left(\mathcal{F}_{\mathrm{b}}(\mathcal{H})\right)$ such that, for all $f \in \mathcal{V}, B e^{i \phi_{T}(f)}=$ $e^{i \phi_{T}(f)} B \cdots(*)$ and $B e^{i \pi_{T}(f)}=e^{i \pi_{T}(f)} B$. Since $T^{-1} \mathcal{V}$ is dense in $\mathcal{H}_{C}$, for each $f \in \mathcal{H}_{C}$, there exists a sequence $\left\{g_{n}\right\}_{n}$ in $\mathcal{V}$ such that $T^{-1} g_{n} \rightarrow f(n \rightarrow \infty)$. By the strong continuity of the mapping: $f \mapsto e^{i \Phi(f)}\left[6\right.$, Theorem X.41-(d)], we have s- $\lim _{n \rightarrow \infty} e^{i \phi_{T}\left(g_{n}\right)}=e^{i \Phi(f)}$, where s- lim means strong limit. By $(*), B e^{i \phi_{T}\left(g_{n}\right)}=e^{i \phi_{T}\left(g_{n}\right)} B$. Taking the strong limit s- $\lim _{n \rightarrow \infty}$ of the both sides, we obtain $B e^{i \Phi(f))}=e^{i \Phi(f)} B$ for all $f \in \mathcal{H}_{C}$. Similarly, using the density of $T \mathcal{V}$ in $\mathcal{H}_{C}$, we can show that, for all $f \in \mathcal{H}_{C}, B e^{i \Pi(f))}=e^{i \Pi(f)} B$. Hence, by the irreducibiliy of $\left\{e^{i \Phi(f)}, e^{i \Pi(f)} \mid f \in \mathcal{H}_{C}\right\}, B=c I$ ( $I$ is identity) with some constant $c \in \mathbb{C}$. Thus $\left\{e^{i \phi_{T}(f)}, e^{i \pi_{T}(f)} \mid f \in \mathcal{V}\right\}$ is irreducible.

It is natural to ask when the Weyl representation $\left(\mathcal{F}_{\mathrm{b}}(\mathcal{H}),\left\{e^{i \phi_{T}(f)}, e^{i \pi_{T}(f)} \mid f \in \mathcal{V}\right\}\right)$ is equivalent or inequivalent to the Fock representation $\left(\mathcal{F}_{\mathrm{b}}(\mathcal{H}),\left\{e^{i \phi(f)}, e^{i \pi(f)} \mid f \in \mathcal{V}\right\}\right)$. As for this problem, we begin with a remark.

We have for all $f \in D(T) \cap D\left(T^{-1}\right)$

$$
\phi_{T}(\Re f)+\pi_{T}(\Im f)=\Phi\left(R_{T} f\right),
$$

with $R_{T} f:=T^{-1} \Re f+i T \Im f$. By direct computation, we have

$$
\Im\left\langle R_{T} f, R_{T} g\right\rangle=\Im\langle f, g\rangle, \quad f, g \in D(T) \cap D\left(T^{-1}\right) .
$$


Hence $R_{T}$ is a symplectic transformation on the symplectic space $(\mathcal{H}, \Im\langle\cdot, \cdot\rangle)$ with domain $D(T) \cap D\left(T^{-1}\right)$ ( $\mathcal{H}$ is considered as a real inner product space with inner product $\Re\langle f, g\rangle, f, g \in \mathcal{H}$.

Suppose that $\mathcal{H}$ is separable, $T \in \mathfrak{B}(\mathcal{H})$ and $T$ is bijective. Let $R_{T}^{\mathrm{t}}$ denote the adjoint of $R_{T}$ as an operator on the real inner product space $(\mathcal{H}, \Re\langle\cdot, \cdot\rangle)$. Then one can apply Shale's theorem [10] to conclude that $\left(\mathcal{F}_{\mathrm{b}}(\mathcal{H}),\left\{e^{i \phi_{T}(f)}, e^{i \pi_{T}(f)} \mid f \in \mathcal{V}\right\}\right)$ is equivalent to $\left(\mathcal{F}_{\mathrm{b}}(\mathcal{H}),\left\{e^{i \phi(f)}, e^{i \pi(f)} \mid f \in \mathcal{V}\right\}\right)$ if and only if $\left(R_{T}^{\mathrm{t}} R_{T}\right)^{1 / 2}-1$ is Hilbert-Schmidt. It is easy to see that $\left(R_{T}^{\mathrm{t}} R_{T}\right)^{1 / 2}-1$ is Hilbert-Schmidt if and only if $T^{2}-1$ is Hilbert-Schmidt. Thus $\left(\mathcal{F}_{\mathrm{b}}(\mathcal{H}),\left\{e^{i \phi_{T}(f)}, e^{i \pi_{T}(f)} \mid f \in \mathcal{V}\right\}\right)$ is equivalent to $\left(\mathcal{F}_{\mathrm{b}}(\mathcal{H}),\left\{e^{i \phi(f)}, e^{i \pi(f)} \mid f \in \mathcal{V}\right\}\right)$ if and only if $T^{2}-1$ is Hilbert-Schmidt.

In the case where $T$ or $T^{-1}$ is unbounded, however, the situation essentially changes, because $R_{T}$ also is unbounded and hence one cannot directly apply Shale's theorem. Thus, in this case, we need to take another route to discuss equivalence or inequivalence of $\left\{\phi_{T}(f), \pi_{T}(f) \mid f \in \mathcal{V}\right\}$ to the Fock representation $\{\phi(f), \pi(f) \mid f \in \mathcal{V}\}$. Blow are the details of a theory for the general case where $T$ or $T^{-1}$ is not necessarily bounded.

It is easy to see that $T\left(T^{2}+1\right)^{-1}$ and $\left(T^{2}-1\right)\left(T^{2}+1\right)^{-1}$ are in $\mathfrak{B}(\mathcal{H})$.

Lemma 4.4 The operator $T+T^{-1}$ is bijective and $\left(T+T^{-1}\right)^{-1} \in \mathfrak{B}(\mathcal{H})$ with

$$
\left(T+T^{-1}\right)^{-1}=T\left(T^{2}+1\right)^{-1} \text {. }
$$

Proof. Let $f \in \operatorname{ker}\left(T+T^{-1}\right)$. Then $T f=-T^{-1} f$. Hence $T f \in D(T)$ and $T^{2} f=-f$, i.e., $\left(T^{2}+1\right) f=0$. Since $T^{2}+1 \geq 1$, it follows that $f=0$. Hence $T+T^{-1}$ is injective.

For each $g \in \mathcal{H}$, let $f=T\left(T^{2}+1\right)^{-1} g$. Then $f \in D(T) \cap D\left(T^{-1}\right)$ and $\left(T+T^{-1}\right) f=g$. Hence $T+T^{-1}$ is surjective. Thus $T+T^{-1}$ is bijective and (4.4) holds.

We introduce a subspace:

$$
\mathcal{V}_{T}:=\left\{\left(T+T^{-1}\right) f \mid f \in \mathcal{V}\right\} .
$$

Lemma 4.5 Assume that $\mathcal{V}_{T}$ is dense and that $\left(\mathcal{F}_{\mathrm{b}}(\mathcal{H}),\left\{e^{i \phi_{T}(f)}, e^{i \pi_{T}(f)} \mid f \in \mathcal{V}\right\}\right)$ has a vacuum. Then $\left(T^{2}-1\right)\left(T^{2}+1\right)^{-1}$ is Hilbert-Schmidt.

Proof. By the assumption, there exists a non-zero vector $\Omega \in \mathcal{F}_{\mathrm{b}}(\mathcal{H})$ such that $\Omega \in$ $D\left(\phi_{T}(f)\right) \cap D\left(\pi_{T}(f)\right)$ for all $f \in \mathcal{V}$ and $\left(\phi_{T}(f)+i \pi_{T}(f)\right) \Omega=0$. Hence, for all $\Psi \in \mathcal{F}_{0}(\mathcal{H})$,

$$
0=\left\langle\left(\phi_{T}(f)-i \pi_{T}(f)\right) \Psi, \Omega\right\rangle=\frac{1}{\sqrt{2}}\left\langle\left(A\left(\left(T+T^{-1}\right) f\right)^{*}-A\left(\left(T-T^{-1}\right) f\right)\right) \Psi, \Omega\right\rangle .
$$

Therefore, for all $g \in \mathcal{V}_{T},\left\langle A(g)^{*} \Psi, \Omega\right\rangle=\left\langle A\left(\left(T^{2}-1\right)\left(T^{2}+1\right)^{-1} g\right) \Psi, \Omega\right\rangle$. This implies that, for all $f \in \mathcal{V}_{T}+i \mathcal{V}_{T}$,

$$
\left\langle A(f)^{*} \Psi, \Omega\right\rangle=\left\langle A\left(C\left(T^{2}-1\right)\left(T^{2}+1\right)^{-1} f\right) \Psi, \Omega\right\rangle .
$$

Hence, by Proposition 3.3, $\left(T^{2}-1\right)\left(T^{2}+1\right)^{-1}$ is Hilbert-Schmidt.

Theorem 4.6 Assume that $\mathcal{H}$ is separable. Suppose that $\left(T^{2}-1\right)\left(T^{2}+1\right)^{-1}$ is not HilbertSchmidt and $\mathcal{V}_{T}$ is dense in $\mathcal{H}_{C}$. Then $\left(\mathcal{F}_{\mathrm{b}}(\mathcal{H}),\left\{e^{i \phi_{T}(f)}, e^{i \pi_{T}(f)} \mid f \in \mathcal{V}\right\}\right)$ is inequivalent to the Fock representation $\left(\mathcal{F}_{\mathrm{b}}(\mathcal{H}),\left\{e^{i \phi(f)}, e^{i \pi(f)} \mid f \in \mathcal{V}\right\}\right)$. 
Proof. Suppose that $\left(\mathcal{F}_{\mathrm{b}}(\mathcal{H}),\left\{e^{i \phi_{T}(f)}, e^{i \pi_{T}(f)} \mid f \in \mathcal{V}\right\}\right)$ is equivalent to the Fock representation $\left(\mathcal{F}_{\mathrm{b}}(\mathcal{H}),\left\{e^{i \phi(f)}, e^{i \pi(f)} \mid f \in \mathcal{V}\right\}\right)$. Then, by Lemma 3.1, $\left(\mathcal{F}_{\mathrm{b}}(\mathcal{H}),\left\{e^{i \phi_{T}(f)}, e^{i \pi_{T}(f)} \mid f \in\right.\right.$ $\mathcal{V}\}$ ) has a vacuum. Hence, by Lemma $4.5,\left(T^{2}-1\right)\left(T^{2}+1\right)^{-1}$ is Hilbert-Schmidt. Thus the desired result follows.

Remark 4.7 Suppose that $T \in \mathfrak{B}(\mathcal{H})$. Then, if $\left(T^{2}-1\right)\left(T^{2}+1\right)^{-1}$ is not Hilbert-Schmidt, then $T^{2}-1$ is not Hilbert-Schmidt and hence the conclusion in Theorem 4.6 holds (see the aforementioned application of Shale's theorem in the case where $T$ is bounded and bijective). Hence, as for a sufficient condition for inequivalence of $\left(\mathcal{F}_{\mathrm{b}}(\mathcal{H}),\left\{\phi_{T}(f), \pi_{T}(f) \mid f \in\right.\right.$ $\mathcal{V}\})$ to the Fock representation $\left(\mathcal{F}_{\mathrm{b}}(\mathcal{H}),\{\phi(f), \pi(f) \mid f \in \mathcal{V}\}\right)$, Theorem 4.6 is certainly an extension of the case where $T$ is bounded and bijective.

For a linear operator $A$ on a Hilbert space, we denote by $\sigma(A)$ and $\left.\sigma_{\mathrm{p}}(A)\right)$ the spectrum and the point spectrum of $A$ respectively.

A sufficient condition for that $\left(T^{2}-1\right)\left(T^{2}+1\right)^{-1}$ is not Hilbert-Schmidt is given by the following lemma (apply it to $S=T^{2}$ ):

Lemma 4.8 Assume that $\mathcal{H}$ is separable with $\operatorname{dim} \mathcal{H}=\infty$. Let $S$ be an injective and non-negative self-adjoint operator on $\mathcal{H}$ satisfying (i) $\sigma_{\mathrm{p}}(S)=\emptyset$ or (ii) $1 \notin \sigma(S)$. Then $(S-1)(S+1)^{-1}$ is not Hilbert-Schmidt.

Proof. Let $K:=(S-1)(S+1)^{-1}$. Then $K$ is a bounded self-adjoint operator on $\mathcal{H}$ and $1-K=2(S+1)^{-1}$. Hence $1-K$ is injective and $S=2(1-K)^{-1}-1$.

Now suppose that $K$ were Hilbert-Schmidt. Then, by the Hilbert-Schmidt theorem, there exists a sequence $\left\{\lambda_{n}\right\}_{n=1}^{\infty}$ of real numbers such that (i) $\left\{\lambda_{n} \mid n \geq 1\right\}=\sigma(K)$; (ii) for all $\lambda_{n} \neq 0, \lambda_{n} \in \sigma_{\mathrm{p}}(K)$ with finite multiplicity; (iii) $\sum_{n=1}^{\infty} \lambda_{n}^{2}<\infty$. Hence, in particular, $\lim _{n \rightarrow \infty} \lambda_{n}=0$. Note that $K \neq 0$. Hence $\sigma_{\mathrm{p}}(K) \backslash\{0\} \neq \emptyset$. By the spectral mapping theorem, for $\lambda_{n} \neq 0$, the number $s_{n}:=2\left(1-\lambda_{n}\right)^{-1}-1 \cdots(*)$ is an eigenvalue of $S$

Assume (i). Then the statement just mentioned yields a contradiction. Therefore, in this case, $K$ is not Hilbert-Schmidt.

Next assume (ii). If $\sigma_{\mathrm{p}}(K) \backslash\{0\}$ is a finite set, then $0 \in \sigma_{\mathrm{p}}(K)$ by $\operatorname{dim} \mathcal{H}=\infty$. Hence $1 \in \sigma_{\mathrm{p}}(S)$. But this is a contradiction.

If $\sigma_{\mathrm{p}}(K) \backslash\{0\}$ is an infinite set, then there exists a subsequence $\left\{\lambda_{n_{k}}\right\}_{k}$ of $\left\{\lambda_{n}\right\}_{n}$ such that, for all $k \geq 1, \lambda_{n_{k}} \neq 0$ and $\lim _{k \rightarrow \infty} \lambda_{n_{k}}=0$. Taking $k \rightarrow \infty$ in (*) with $n$ replaced by $n_{k}$, we obtain $\lim _{k \rightarrow \infty} s_{n_{k}}=1$. Hence $1 \in \sigma(S)$. But this also is a contradiction. Thus $K$ is not Hilbert-Schmidt.

Combining Theorem 4.6 with Lemma 4.8, we obtain the following result:

Corollary 4.9 Assume that $\mathcal{H}$ is separable with $\operatorname{dim} \mathcal{H}=\infty$ and $\mathcal{V}_{T}$ is dense in $\mathcal{H}_{C}$. Suppose that $\sigma_{\mathrm{p}}\left(T^{2}\right)=\emptyset$ or $1 \notin \sigma\left(T^{2}\right)$. Then $\left(\mathcal{F}_{\mathrm{b}}(\mathcal{H}),\left\{e^{i \phi_{T}(f)}, e^{i \pi_{T}(f)} \mid f \in \mathcal{V}\right\}\right)$ is inequivalent to the Fock representation $\left(\mathcal{F}_{\mathbf{b}}(\mathcal{H}),\left\{e^{i \phi(f)}, e^{i \pi(f)} \mid f \in \mathcal{V}\right\}\right)$.

Proof. By Lemma 4.8 with $S=T^{2},\left(T^{2}-1\right)\left(T^{2}+1\right)^{-1}$ is not Hilbert-Schmidt. Hence the desired result follows from Theorem 4.6. 


\section{Main Theorems}

We now state main theorems in the present paper.

Theorem 5.1 Assume that $\mathcal{H}$ is separable. Let $T_{1}, T_{2} \in \mathrm{S}_{\mathcal{V}}$ such that the following (a) and (b) hold:

(a) $D\left(T_{1}^{-1} T_{2}^{2} T_{1}^{-1}\right) \cap D\left(T_{1} T_{2}^{-2} T_{1}\right)$ and $D\left(T_{2}^{-1} T_{1}^{2} T_{2}^{-1}\right) \cap D\left(T_{2} T_{1}^{-2} T_{2}\right)$ are dense in $\mathcal{H}$.

(b) $T_{2}^{-1} T_{1}$ and $T_{2} T_{1}^{-1}$ are bounded with $\mathcal{V} \subset D\left(T_{2}^{-1} T_{1}\right) \cap D\left(T_{2} T_{1}^{-1}\right) \cap D\left(T_{1}^{-1} T_{2}\right) \cap$ $D\left(T_{1} T_{2}^{-1}\right)$.

Then $\left(\mathcal{F}_{\mathrm{b}}(\mathcal{H}),\left\{e^{i \phi_{T_{1}}(f)}, e^{i \pi_{T_{1}}(f)} \mid f \in \mathcal{V}\right\}\right)$ is equivalent to $\left(\mathcal{F}_{\mathrm{b}}(\mathcal{H}),\left\{e^{i \phi_{T_{2}}(f)}, e^{i \pi_{T_{2}}(f)} \mid f \in \mathcal{V}\right\}\right)$ if and only if $\overline{T_{2}^{-1} T_{1}-T_{2} T_{1}^{-1}}$ is Hilbert-Schmidt.

Remark 5.2 We want to emphasize that, in Theorem 5.1, $T_{1}$ and $T_{2}$ are not necessarily commuting.

Remark 5.3 The contraposition of Theorem 5.1 yields a necessary and sufficient condition for $\left(\mathcal{F}_{\mathrm{b}}(\mathcal{H}),\left\{e^{i \phi_{T_{1}}(f)}, e^{i \pi_{T_{1}}(f)} \mid f \in \mathcal{V}\right\}\right)$ and $\left(\mathcal{F}_{\mathrm{b}}(\mathcal{H}),\left\{e^{i \phi_{T_{2}}(f)}, e^{i \pi_{T_{2}}(f)} \mid f \in \mathcal{V}\right\}\right)$ to be inequivalent.

Remark 5.4 The conditions for $T_{1}$ and $T_{2}$ in Theorem 5.1 are related to an equivalence relation in a subset of $\mathcal{S}_{\mathcal{V}}$. Let

$$
\mathcal{S}_{\mathcal{V}}^{\times}:=\left\{T \in \mathcal{S}_{\mathcal{V}} \mid T \text { is surjective }\right\} .
$$

Then, for all $T \in \mathcal{S}_{\mathcal{V}}^{\times}, T^{-1} \in \mathfrak{B}(\mathcal{H})$. For $T_{1}, T_{2} \in \mathcal{S}_{\mathcal{V}}^{\times}$, we write $T_{1} \sim T_{2}$ if the following conditions (i) and (ii) are satisfied: (i) $T_{2} T_{1}^{-1}, T_{1} T_{2}^{-1} \in \mathfrak{B}(\mathcal{H})$; (ii) $\overline{T_{2}^{-1} T_{1}-T_{2} T_{1}^{-1}}$ is HilbertSchmidt. It is easy to see that the relation $\sim$ is an equivalent relation in $\mathcal{S}_{\mathcal{V}}^{\times}$. Hence Theorem 5.1 implies the following: Let $T_{1}, T_{2} \in \mathcal{S}_{\mathcal{V}}^{\times}$. Then $\rho_{T_{1}}:=\left(\mathcal{F}_{\mathrm{b}}(\mathcal{H}),\left\{e^{i \phi_{T_{1}}(f)}, e^{i \pi_{T_{1}}(f)} \mid f \in\right.\right.$ $\mathcal{V}\})$ is equivalent to $\rho_{T_{2}}:=\left(\mathcal{F}_{\mathrm{b}}(\mathcal{H}),\left\{e^{i \phi_{T_{2}}(f)}, e^{i \pi_{T_{2}}(f)} \mid f \in \mathcal{V}\right\}\right)$ if and only if $T_{1} \sim T_{2}$ and condition (a) holds. Hence, for a subset $\mathcal{S}_{\mathcal{V}, 0}$ of $\mathcal{S}_{\mathcal{V}}^{\times}$such that (a) holds for all $T_{1}, T_{2} \in \mathcal{S}_{\mathcal{V}, 0}$, $\rho_{T_{1}}$ is equivalent to $\rho_{T_{2}}$ if and only if $T_{1} \sim T_{2}$. This is an interesting phenomenon to note.

In the case where at least one of $T_{2}^{-1} T_{1}$ and $T_{2} T_{1}^{-1}$ is unbounded, the proof of Theorem 5.1 given below is not valid any more. In this case, we need a separate consideration. To state a theorem in such a case, we need a lemma.

Lemma 5.5 For all $T_{1}, T_{2} \in \mathrm{S}_{\mathcal{V}}$,

$$
T_{+}:=T_{2}^{-1} T_{1}+T_{2} T_{1}^{-1}
$$

is injective.

Proof. Let $f \in \operatorname{ker} T_{+}$. Then $f \in D\left(T_{2}^{-1} T_{1}\right) \cap D\left(T_{2} T_{1}^{-1}\right)$ and $T_{2}^{-1} T_{1} f=-T_{2} T_{1}^{-1} f$. Hence $T_{1} f=-T_{2}^{2} T_{1}^{-1} f$. Therefore $f=-T_{1}^{-1} T_{2}^{2} T_{1}^{-1} f$. Taking the inner product of both sides with $f$, we have $\|f\|^{2}=-\left\langle T_{1}^{-1} f, T_{2}^{2} T_{1}^{-1} f\right\rangle \leq 0$. Hence $\|f\|^{2}=0$, implying that $f=0$. Thus $T_{+}$is injective.

Let

$$
T_{-}:=T_{2}^{-1} T_{1}-T_{2} T_{1}^{-1}
$$


Theorem 5.6 Assume that $\mathcal{H}$ is separable. Let $T_{1}, T_{2} \in \mathrm{S}_{\mathcal{V}}$ such that the following (a) -(c) hold:

(a) $\left(T_{1} \mathcal{V}\right) \cap\left(T_{1}^{-1} \mathcal{V}\right)$ is dense in $\mathcal{H}_{C}$.

(b) $\left\{T_{+} f \mid f \in\left(T_{1} \mathcal{V}\right) \cap\left(T_{1}^{-1} \mathcal{V}\right)\right\}$ is dense in $\mathcal{H}_{C}$.

(c) $T_{-} T_{+}^{-1}$ is bounded and its closure $\overline{T_{-} T_{+}^{-1}}$ is not Hilbert-Schmidt.

Then $\left(\mathcal{F}_{\mathrm{b}}(\mathcal{H}),\left\{e^{i \phi_{T_{1}}(f)}, e^{i \pi_{T_{1}}(f)} \mid f \in \mathcal{V}\right\}\right)$ is inequivalent to $\left(\mathcal{F}_{\mathrm{b}}(\mathcal{H}),\left\{e^{i \phi_{T_{2}}(f)}, e^{i \pi_{T_{2}}(f)} \mid f \in\right.\right.$ $\mathcal{V}\})$.

Remark 5.7 In Theorem 5.6, $T_{2}^{-1} T_{1}$ and $T_{2} T_{1}^{-1}$ are not necessarily bounded.

\subsection{Proof of Theorem $\mathbf{5 . 1}$}

We prove Theorem 5.1 by a series of lemmas.

Lemma 5.8 Let $T_{1}, T_{2} \in \mathrm{S}_{\mathcal{V}}(\mathcal{H})$ such that $D\left(T_{2}^{-1} T_{1}\right) \cap D\left(T_{2} T_{1}^{-1}\right)$ is dense in $\mathcal{H}$. Let

$$
S_{ \pm}:=\frac{1}{2} T_{ \pm}=\frac{1}{2}\left(T_{2}^{-1} T_{1} \pm T_{2} T_{1}^{-1}\right) .
$$

Then

(i)

$$
\begin{aligned}
& S_{+}^{*} S_{+}-S_{-}^{*} S_{-}=I, \\
& S_{-}^{*} S_{+}=S_{+}^{*} S_{-}
\end{aligned}
$$

on $D\left(T_{1}^{-1} T_{2}^{2} T_{1}^{-1}\right) \cap D\left(T_{1} T_{2}^{-2} T_{1}\right)$.

(ii)

$$
\begin{aligned}
& S_{+} S_{+}^{*}-S_{-} S_{-}^{*}=I, \\
& S_{-} S_{+}^{*}=S_{+} S_{-}^{*}
\end{aligned}
$$

on $D\left(T_{2}^{-1} T_{1}^{2} T_{2}^{-1}\right) \cap D\left(T_{2} T_{1}^{-2} T_{2}\right)$.

Proof. (i) By the present assumption, $S_{ \pm}$are densely defined. It follows that

$$
S_{ \pm}^{*} \supset \frac{1}{2}\left(T_{1} T_{2}^{-1} \pm T_{1}^{-1} T_{2}\right)
$$

Let $\Psi \in D\left(T_{1}^{-1} T_{2}^{2} T_{1}^{-1}\right) \cap D\left(T_{1} T_{2}^{-2} T_{1}\right)$. Then $\Psi \in D\left(S_{+}^{*} S_{+}\right) \cap D\left(S_{-}^{*} S_{-}\right)$and

$$
\begin{aligned}
& S_{+}^{*} S_{+} \Psi=\frac{1}{4}\left(T_{1} T_{2}^{-2} T_{1}+2+T_{1}^{-1} T_{2}^{2} T_{1}^{-1}\right) \Psi, \\
& S_{-}^{*} S_{-} \Psi=\frac{1}{4}\left(T_{1} T_{2}^{-2} T_{1}-2+T_{1}^{-1} T_{2}^{2} T_{1}^{-1}\right) \Psi .
\end{aligned}
$$


Hence $S_{+}^{*} S_{+} \Psi-S_{-}^{*} S_{-} \Psi=\Psi$. Thus (5.4) holds on $D\left(T_{1}^{-1} T_{2}^{2} T_{1}^{-1}\right) \cap D\left(T_{1} T_{2}^{-2} T_{1}\right)$. Similarly one can prove (5.5).

(ii) This also can be proved by direct computations as in (i).

Relations (5.4)-(5.7) remind us the theory of Bogoliubov transformations (e.g., [7, Theorem XI.108]). Thus we are led to proceed as follows.

Suppose that the assumption of Theorem 5.1 is satisfied. By conditions (a) and (b) in Theorem 5.1, $S_{ \pm}$are densely defined and bounded. We denote their closure by $\bar{S}_{ \pm}$. Then (5.4)-(5.7) can be extended to the case $S_{ \pm}$replaced by $\bar{S}_{ \pm}$.

For each $f \in \mathcal{H}$, we introduce a new operator $B(f)$ by

$$
B(f):=A\left(C \bar{S}_{-} f\right)^{*}+A\left(\bar{S}_{+} f\right), \quad f \in \mathcal{H} .
$$

Hence

$$
B(f)^{*} \supset A\left(\bar{S}_{+} f\right)^{*}+A\left(C \bar{S}_{-} f\right), \quad f \in \mathcal{H} .
$$

Note that $\left\{B(f), B(f)^{*} \mid f \in \mathcal{H}\right\}$ satisfy

$$
\begin{aligned}
& {\left[B(f), B(g)^{*}\right]=\langle f, g\rangle,} \\
& {[B(f), B(g)]=0, \quad\left[B(f)^{*}, B(g)^{*}\right]=0, \quad f, g \in \mathcal{H},}
\end{aligned}
$$

on $\mathcal{F}_{0}(\mathcal{H})$. Hence the correspondence $\left(A(\cdot), A(\cdot)^{*}\right) \mapsto\left(B(\cdot), B(\cdot)^{*}\right)$ is a Bogoliubov transformation.

Lemma 5.9 Under the same assumption as in Theorem 5.1, there exists a unitary operator $U$ on $\mathcal{F}_{\mathrm{b}}(\mathcal{H})$ such that

$$
\overline{B(f)}=U A(f) U^{-1}, \quad f \in \mathcal{H}
$$

if and only if $\bar{S}_{-}$is Hilbert-Schmidt.

Proof. This follows from an application of [7, Theorem XI.108].

We are now ready to show the sufficiency of the condition that $\overline{T_{2}^{-1} T_{1}-T_{2} T_{1}^{-1}}$ is Hilbert-Schmidt in Theorem 5.1:

Lemma 5.10 Under the same assumption as in Theorem 5.1, suppose that $\overline{T_{2}^{-1} T_{1}-T_{2} T_{1}^{-1}}$ is Hilbert-Schmidt. Then there exists a unitary operator $U$ on $\mathcal{F}_{\mathrm{b}}(\mathcal{H})$ such that operator equations

$$
\begin{aligned}
& U \phi_{T_{1}}(f) U^{-1}=\phi_{T_{2}}(f), \\
& U \pi_{T_{1}}(f) U^{-1}=\pi_{T_{2}}(f), \quad f \in \mathcal{V},
\end{aligned}
$$

hold. Namely, $\left(\mathcal{F}_{\mathrm{b}}(\mathcal{H}),\left\{e^{i \phi_{T_{1}}(f)}, e^{i \pi_{T_{1}}(f)}\right\}\right)$ is equivalent to $\left(\mathcal{F}_{\mathbf{b}}(\mathcal{H}),\left\{e^{i \phi_{T_{2}}(f)}, e^{i \pi_{T_{2}}(f)}\right\}\right)$.

Proof. By the present assumption $\bar{S}_{-}$is Hilbert-Schmidt. Hence, by Lemma 5.9, there exists a unitary operator $U$ on $\mathcal{F}_{\mathrm{b}}(\mathcal{H})$ satisfying (5.8). This implies also

$$
B(f)^{*}=U A(f)^{*} U^{-1}, \quad f \in \mathcal{H},
$$


where we have used the fact that, for any closable operator $T, T^{*}=(\bar{T})^{*}$. Hence, for all $f \in \mathcal{H}$, we have

$$
\frac{B(f)^{*} \pm \overline{B(f)}}{\sqrt{2}}=U \frac{1}{\sqrt{2}}\left(A(f)^{*} \pm A(f)\right) U^{-1}
$$

On the other hand, it is easy to see that, for all $\Psi \in \mathcal{F}_{0}(\mathcal{H})$ and $f \in D\left(T_{2}^{-1} T_{1}\right) \cap$ $D\left(T_{2} T_{1}^{-1}\right) \cap \mathcal{H}_{C}$,

$$
\frac{1}{\sqrt{2}}\left(B(f)^{*}+B(f)\right) \Psi=\phi_{T_{2}}\left(T_{1} f\right) \Psi, \quad \frac{i}{\sqrt{2}}\left(B(f)^{*}-B(f)\right)=\pi_{T_{2}}\left(T_{1}^{-1} f\right) \Psi .
$$

Hence, for all $f \in D\left(T_{2}^{-1} T_{1}\right) \cap D\left(T_{2} T_{1}^{-1}\right) \cap \mathcal{H}_{C}$,

$$
\phi_{T_{2}}\left(T_{1} f\right) \Psi=U \phi(f) U^{-1} \Psi, \pi_{T_{2}}\left(T_{1}^{-1} f\right) \Psi=U \pi(f) U^{-1} \Psi, \Psi \in \mathcal{F}_{0}(\mathcal{H}),
$$

which, by condition (b), imply the following equations:

$$
\begin{aligned}
& \phi_{T_{2}}(f) \Psi=U \phi_{T_{1}}(f) U^{-1} \Psi, \\
& \pi_{T_{2}}(f) \Psi=U \pi_{T_{1}}(f) U^{-1} \Psi, \quad f \in \mathcal{V}, \Psi \in \mathcal{F}_{0}(\mathcal{H}) .
\end{aligned}
$$

Hence

$$
\phi_{T_{2}}(f) \uparrow \mathcal{F}_{0}(\mathcal{H}) \subset U \phi_{T_{1}}(f) U^{-1}, \quad \pi_{T_{2}}(f) \uparrow \mathcal{F}_{0}(\mathcal{H}) \subset U \pi_{T_{1}}(f) U^{-1}, \quad f \in \mathcal{V} .
$$

Recall that $\mathcal{F}_{0}(\mathcal{H})$ is a core for $\phi_{T_{2}}(f)$ and $\pi_{T_{2}}(f)$. Moreover, $U \phi_{T_{1}}(f) U^{-1}$ and $U \pi_{T_{1}}(f) U^{-1}$ are self-adjoint. Hence operator equations (5.9) and (5.10) follow.

We next show the necessity of the condition that $\overline{T_{2}^{-1} T_{1}-T_{2} T_{1}^{-1}}$ is Hilbert-Schmidt in Theorem 5.1.

Using (3.1), one can easily prove the following lemma.

Lemma 5.11 Let $\mathcal{V}$ be a dense subspace in $\mathcal{H}_{C}$. Then $\{g \in \mathcal{H} \mid\langle f, g\rangle=0, f \in \mathcal{V}\}=\{0\}$.

Lemma 5.12 Under the same assumption as in Theorem 5.1, suppose that there exists a unitary operator $U$ on $\mathcal{F}_{\mathrm{b}}(\mathcal{H})$ satisfying (5.9) and (5.10). Then $\overline{T_{2}^{-1} T_{1}-T_{2} T_{1}^{-1}}$ is HilbertSchmidt.

Proof. It is easy to see that, for all $\Psi \in \mathcal{F}_{0}(\mathcal{H})$ and $f \in \mathcal{V}$,

$$
A(f) \Psi=\frac{1}{\sqrt{2}}\left(\phi_{T_{1}}\left(T_{1} f\right)+i \pi_{T_{1}}\left(T_{1}^{-1} f\right)\right) \Psi .
$$

Hence

$$
A(f) \Psi=\frac{1}{\sqrt{2}}\left(U^{-1} \phi_{T_{2}}\left(T_{1} f\right) U \Psi+i U^{-1} \pi_{T_{2}}\left(T_{1}^{-1} f\right) U \Psi\right)
$$

which implies that

$$
U A(f) \Psi=\frac{1}{\sqrt{2}}\left(\phi_{T_{2}}\left(T_{1} f\right) U \Psi+i \pi_{T_{2}}\left(T_{1}^{-1} f\right) U \Psi\right) .
$$


Hence, for all $\Phi \in \mathcal{F}_{0}(\mathcal{H})$,

$$
\left\langle U^{-1} \Phi, A(f) \Psi\right\rangle=\left\langle U^{-1}\left(A\left(S_{+} f\right)^{*} \Phi+A\left(S_{-} f\right) \Phi\right), \Psi\right\rangle .
$$

Since $\mathcal{F}_{0}(\mathcal{H})$ is a core for $A(f)$, it follows that $U^{-1} \Phi \in D\left(A(f)^{*}\right)$ and $A(f)^{*} U^{-1} \Phi=$ $U^{-1}\left(A\left(S_{+} f\right)^{*}+A\left(S_{-} f\right)\right) \Phi$. By the density of $\mathcal{V}$ in $\mathcal{H}_{C}$ and Lemma 2.1, this equation can be extended to all $f \in \mathcal{H}_{C}$ with $S_{ \pm}$replaced by $\bar{S}_{ \pm}$:

$$
A(f)^{*} U^{-1} \Phi=U^{-1}\left(A\left(\bar{S}_{+} f\right)^{*}+A\left(\bar{S}_{-} f\right)\right) \Phi, \quad f \in \mathcal{H}_{C} .
$$

By (2.5), we have $\left\langle A(f)^{*} U^{-1} \Phi, \Omega_{\mathrm{F}}\right\rangle=0$. Hence, putting $\Omega:=U \Omega_{\mathrm{F}}$, we obtain from $(5.13)$

$$
\left\langle A\left(\bar{S}_{+} f\right)^{*} \Phi, \Omega\right\rangle=-\left\langle A\left(\bar{S}_{-} f\right) \Phi, \Omega\right\rangle, \quad f \in \mathcal{H}_{C} .
$$

It follows from (5.4) that

$$
\bar{S}_{+}^{*} \bar{S}_{+}=1+\bar{S}_{-}^{*} \bar{S}_{-} .
$$

This implies that $\bar{S}_{+}^{*} \bar{S}_{+}$is bijective on $\mathcal{H}_{C}$ (note that $\bar{S}_{ \pm}$are reduced by $\mathcal{H}_{C}$ ). In particular, $\bar{S}_{+}$is injective on $\mathcal{H}_{C}$. On the other hand, (5.6) gives

$$
\bar{S}_{+} \bar{S}_{+}^{*}=1+\bar{S}_{-} \bar{S}_{-}^{*} .
$$

This implies that $\bar{S}_{+} \bar{S}_{+}^{*}$ is bijective on $\mathcal{H}_{C}$. In particular, $\bar{S}_{+}$is surjective on $\mathcal{H}_{C}$. Hence $\bar{S}_{+}$is bijective on $\mathcal{H}_{C}$. Hence, putting $K:=\bar{S}_{-} \bar{S}_{+}^{-1}$, we have for all $f \in \mathcal{H}_{C},\left\langle A(f)^{*} \Phi, \Omega\right\rangle=$ $-\langle A(K f) \Phi, \Omega\rangle$. By using (3.1), this equation can be extended to

$$
\left\langle A(f)^{*} \Phi, \Omega\right\rangle=-\langle A(C K f) \Phi, \Omega\rangle, \quad f \in \mathcal{H} .
$$

Hence, by Proposition 3.3, $K$ is Hilbert-Schmidt. Thus $\bar{S}_{-}=K \bar{S}_{+}$is Hilbert-Schmidt.

This completes a proof of Theorem 5.1.

\subsection{Proof of Theorem 5.6}

Suppose that $\left(\mathcal{F}_{\mathrm{b}}(\mathcal{H}),\left\{e^{i \phi_{T_{1}}(f)}, e^{i \pi_{T_{1}}(f)} \mid f \in \mathcal{V}\right\}\right)$ were equivalent to $\left(\mathcal{F}_{\mathrm{b}}(\mathcal{H}),\left\{e^{i \phi_{T_{2}}(f)}\right.\right.$, $\left.\left.e^{i \pi_{T_{2}}(f)} \mid f \in \mathcal{V}\right\}\right)$. Then there exists a unitary operator $U$ on $\mathcal{F}_{\mathrm{b}}(\mathcal{H})$ such that $(5.9)$ and $(5.10)$ hold. Let $\mathcal{R}:=\left(T_{1} \mathcal{V}\right) \cap\left(T_{1}^{-1} \mathcal{V}\right)$. Hence, for all $g \in \mathcal{R}$,

$$
U^{-1} \phi_{T_{2}}\left(T_{1} g\right) U=\phi(g), \quad U^{-1} \pi_{T_{2}}\left(T_{1}^{-1} g\right) U=\pi(g) .
$$

Using these equations, in the same manner as in the proof of Theorem 4.6, one can show that, for all $g \in \in \mathcal{R}$ and $\Phi \in \mathcal{F}_{0}(\mathcal{H})$,

$$
\left\langle A\left(T_{+} g\right)^{*} \Phi, \Psi_{0}\right\rangle=-\left\langle A\left(T_{-} g\right) \Phi, \Psi_{0}\right\rangle,
$$

where $\Psi_{0}:=U \Omega_{\mathrm{F}}$. By Lemma 5.5, for all $f \in T_{+} \mathcal{R},\left\langle A(f)^{*} \Phi, \Psi_{0}\right\rangle=-\left\langle A\left(T_{-} T_{+}^{-1} f\right) \Phi, \Psi_{0}\right\rangle$. Hence $\left\langle A(f)^{*} \Phi, \Psi_{0}\right\rangle=-\left\langle A\left(C T_{-} T_{+}^{-1} f\right) \Phi, \Psi_{0}\right\rangle$ for all $f \in T_{+} \mathcal{R}+i T_{+} \mathcal{R}$. By condition (b) in Theorem 5.6, $T_{+} \mathcal{R}+i T_{+} \mathcal{R}$ is dense in $\mathcal{H}$. Hence, by Proposition 3.3, $\overline{T_{-} T_{+}^{-1}}$ is HilbertSchmidt. But this contradicts the present assumption. Thus $\left(\mathcal{F}_{\mathrm{b}}(\mathcal{H}),\left\{e^{i \phi_{T_{1}}(f)}, e^{i \pi_{T_{1}}(f)}\right.\right.$ $\mid f \in \mathcal{V}\})$ is inequivalent to $\left(\mathcal{F}_{\mathrm{b}}(\mathcal{H}),\left\{e^{i \phi_{T_{2}}(f)}, e^{i \pi_{T_{2}}(f)} \mid f \in \mathcal{V}\right\}\right)$. 


\section{Applications}

\subsection{Inequivalence of time-zero field and conjugate momentum for different masses}

We now show that Theorem 1.1 is a consequence of an application of Theorem 5.1.

Let

$$
\rho_{m}:=\left(\mathcal{F}_{\mathrm{b}}\left(L^{2}\left(\mathbb{R}^{d}\right)\right),\left\{e^{i \phi_{m}(f)}, e^{i \pi_{m}(f)} \mid f \in \mathcal{V}_{d, m}\right\}\right)
$$

be the Weyl representation of CCR in Example 2.6 with $m>0$ and $C_{d}$ be the complex conjugation on $L^{2}\left(\mathbb{R}^{d}\right)$ :

$$
\left(C_{d} f\right)(x)=f(x)^{*}, \quad f \in L^{2}\left(\mathbb{R}^{d}\right), \text { a.e. } x \in \mathbb{R}^{d} .
$$

The Fourier transform of $C_{d}$

$$
\hat{C}_{d}:=\mathcal{F}_{d} C_{d} \mathcal{F}_{d}^{-1}
$$

is a conjugation on $L^{2}\left(\mathbb{R}^{d *}\right)$ with

$$
\left(\hat{C}_{d} g\right)(k)=g(-k)^{*}, \quad g \in L^{2}\left(\mathbb{R}^{d *}\right), \text { a.e. } k \in\left(\mathbb{R}^{d}\right)^{*} .
$$

The real Hilbert space associated with $\hat{C}_{d}$ is given by

$$
L^{2}\left(\mathbb{R}^{d *}\right)_{\hat{C}_{d}}=\left\{g \in L^{2}\left(\mathbb{R}^{d *}\right) \mid \hat{C}_{d} g=g\right\} .
$$

We have

$$
\begin{aligned}
& \mathcal{F}_{d} \mathcal{S}_{\mathbb{R}}\left(\mathbb{R}^{d}\right)=\mathcal{S}\left(\mathbb{R}^{d *}\right)_{\hat{C}_{d}}:=\left\{g \in \mathcal{S}\left(\mathbb{R}^{d *}\right) \mid \hat{C}_{d} g=g\right\} \\
& \mathcal{F}_{d} L_{\mathbb{R}}^{2}\left(\mathbb{R}^{d}\right)=L^{2}\left(\mathbb{R}^{d *}\right)_{\hat{C}_{d}} .
\end{aligned}
$$

Lemma 6.1 Let $m \geq 0$. Then:

(i) $C_{d} h_{m} \subset h_{m} C_{d}$.

(ii) $\mathcal{V}_{d, m} \subset D\left(h_{m}\right) \cap D\left(h_{m}^{-1}\right)$.

(iii) $h_{m} \mathcal{V}_{d, m}$ and $h_{m}^{-1} \mathcal{V}_{d, m}$ are dense in $L_{\mathbb{R}}^{2}\left(\mathbb{R}^{d}\right)$.

(iv) $\left\{\left(h_{m}+h_{m}^{-1}\right) f \mid f \in \mathcal{V}_{d, m}\right\}$ is dense in $L_{\mathbb{R}}^{2}\left(\mathbb{R}^{d}\right)$.

Proof. (i) Let $f \in D\left(h_{m}\right)$. Then $\widehat{C_{d} f}(k)=\hat{f}(-k)^{*}$. Hence $\int_{\mathbb{R}^{d *}}\left|\omega_{m}(k) \widehat{C_{d} f}(k)\right|^{2} d k=$ $\int_{\mathbb{R}^{d *}}\left|\omega_{m}(k) \hat{f}(k)\right|^{2} d k<\infty$. Hence $C_{d} f \in D\left(h_{m}\right)$. Moreover,

$$
\left(\mathcal{F}_{d}\left(h_{m} C_{d} f\right)\right)(k)=\omega_{m}(k) \hat{f}(-k)^{*}=\left(\omega_{m}(-k) \hat{f}(-k)\right)^{*}=\left(\mathcal{F}_{d}\left(C_{d} h_{m} f\right)\right)(k), \quad \text { a.e. } k \in \mathbb{R}^{d *} .
$$

Hence $h_{m} C_{d} f=C_{d} h_{m} f$. Thus $C_{d} h_{m} \subset h_{m} C_{d}$.

(ii) This is obvious from the definition of $\mathcal{V}_{d, m}$.

(iii) It is easy to see that $\left\{\omega_{m}^{ \pm 1} \hat{f} \mid f \in \mathcal{V}_{d, m}\right\}$ are dense in $L^{2}\left(\mathbb{R}^{d *}\right)_{\hat{C}_{d}}$. Hence $h_{m}^{ \pm 1} \mathcal{V}_{d, m}$ are dense in $L_{\mathbb{R}}^{2}\left(\mathbb{R}^{d}\right)$. 
(iv) This follows from the fact that $\left\{\left(\omega_{m}+\omega_{m}^{-1}\right) \hat{f} \mid f \in \mathcal{V}_{d, m}\right\}$ is dense in $L^{2}\left(\mathbb{R}^{d *}\right)_{\hat{C}_{d}}$.

By using Lemma 6.1, one can see that $\rho_{m}$ is a special case of the Weyl representation $\left(\mathcal{F}_{\mathrm{b}}(\mathcal{H}),\left\{e^{i \phi_{T}(f)}, e^{i \pi_{T}(f)} \mid f \in \mathcal{V}\right\}\right)$ in Lemma 4.3 with the following choice of $(\mathcal{H}, C, T, \mathcal{V})$ :

$$
\mathcal{H}=L^{2}\left(\mathbb{R}^{d}\right), C=C_{d}, T=h_{m}^{1 / 2}, \mathcal{V}=\mathcal{V}_{d, m}
$$

We first note the following fact:

Theorem 6.2 For all $m \geq 0, \rho_{m}$ has no vacuum.

Proof. It is easy to see that $\sigma_{\mathrm{p}}\left(h_{m}\right)=\sigma_{\mathrm{p}}\left(\omega_{m}\right)=\emptyset$. Hence, by Corollary $4.9, \rho_{m}$ has no vacuum.

Lemma 6.3 Let $m_{1}, m_{2}>0$. The following operators are all bounded:

$$
h_{m_{1}}^{-1 / 2} h_{m_{2}} h_{m_{1}}^{-1 / 2}, h_{m_{1}}^{1 / 2} h_{m_{2}}^{-1} h_{m_{1}}^{1 / 2}, h_{m_{2}}^{-1 / 2} h_{m_{1}}^{1 / 2}, h_{m_{2}}^{1 / 2} h_{m_{1}}^{-1 / 2} .
$$

Proof. This can be proved by the functional calculus for the self-adjoint operator $\Delta$ or using the Fourier transform.

By Lemma 6.3, conditions (a) and (b) in Theorem 5.1 are satisfied with $T_{1}=h_{m_{1}}^{1 / 2}$ and $T_{2}=h_{m_{2}}^{1 / 2}$. It is easy to see that

$$
\overline{h_{m_{2}}^{-1 / 2} h_{m_{1}}^{1 / 2}-h_{m_{2}}^{1 / 2} h_{m_{1}}^{-1 / 2}}=\left(m_{1}^{2}-m_{2}^{2}\right)\left(h_{m_{1}}+h_{m_{2}}\right)^{-1} h_{m_{1}}^{-1 / 2} h_{m_{2}}^{-1 / 2} .
$$

We have

$$
\mathcal{F}_{d}\left(h_{m_{1}}+h_{m_{2}}\right)^{-1} h_{m_{1}}^{-1 / 2} h_{m_{2}}^{-1 / 2} \mathcal{F}_{d}^{-1}=\left(\omega_{m_{1}}+\omega_{m_{2}}\right)^{-1} \omega_{m_{1}}^{-1 / 2} \omega_{m_{2}}^{-1 / 2} .
$$

This operator is a non-zero multiplication operator and hence it is not Hilbert-Schmidt. Hence $\overline{h_{m_{2}}^{-1 / 2} h_{m_{1}}^{1 / 2}-h_{m_{2}}^{1 / 2} h_{m_{1}}^{-1 / 2}}$ is Hilbert-Schmidt if and only if $m_{1}=m_{2}$. Thus, by Theorem 5.1, we obtain the following result:

Theorem 6.4 Let $m_{1}, m_{2}>0$. Then $\rho_{m_{1}}$ and $\rho_{m_{2}}$ are inequivalent if and only if $m_{1} \neq$ $m_{2}$.

We next consider the case where one of $m_{1}$ and $m_{2}$ is zero, say, $m_{2}=0$. In this case, $h_{m_{1}} h_{0}^{-1}$ is unbounded. Hence we can not immediately apply Theorem 5.1. But the idea of the proof of Theorem 5.1 is used. We have the following result:

Theorem 6.5 Let $m>0$. Then $\rho_{m}$ is inequivalent to $\rho_{0}$.

Proof. Suppose that $\rho_{m}$ were equivalent to $\rho_{0}$. Then there exists a unitary operator $U$ on $\mathcal{F}_{\mathrm{b}}\left(L^{2}\left(\mathbb{R}^{d}\right)\right)$ such that

$$
U \phi_{m}(f) U^{-1}=\phi_{0}(f), \quad U \pi_{m}(f) U^{-1}=\pi_{0}(f), \quad f \in \mathcal{V}_{d, 0} .
$$

Hence

$$
U \phi_{T}(g) U^{-1}=\phi(g), \quad U \pi_{T}(g) U^{-1}=\pi(g), \quad g \in \mathcal{D}_{0},
$$


where $T=h_{m}^{1 / 2} h_{0}^{-1 / 2}$ and

$$
\mathcal{D}_{0}:=\left(h_{0}^{-1 / 2} \mathcal{V}_{d, 0}\right) \cap\left(h_{0}^{1 / 2} \mathcal{V}_{d, 0}\right)
$$

It is easy to see that $\sigma_{\mathrm{p}}\left(T^{2}\right)=\emptyset$. Hence, by Lemma $4.8,\left(T^{2}-1\right)\left(T^{2}+1\right)^{-1}$ is not HilbertSchmidt. This contradicts Theorem 4.6. Thus $\rho_{m}$ is inequivalent to $\rho_{0}$.

Remark 6.6 An alternative proof of Theorem 6.5 is given by an application of Theorem 5.6 .

\subsection{A more general family of inequivalent representations of CCR on $\mathcal{F}_{\mathrm{b}}\left(L^{2}\left(\mathbb{R}^{d}\right)\right)$}

The representation $\rho_{m}$ can be regarded as a special case of a more general representation of CCR on $\mathcal{F}_{\mathrm{b}}\left(L^{2}\left(\mathbb{R}^{d}\right)\right)$. Let $v$ be a real-valued Borel measurable function on $\mathbb{R}^{d *}$ such that

$$
v(k)=v(-k), \quad 0<|v(k)|<\infty, \quad \text { a.e. } k \in \mathbb{R}^{d *} .
$$

For each $j=1, \ldots, d$, we denote by $D_{j}$ the generalized partial differential operator in the variable $x_{j}$ acting in $L^{2}\left(\mathbb{R}^{d}\right)$ and set $\nabla:=\left(-i D_{1}, \ldots,-i D_{d}\right)$. Let

$$
v(-i \nabla):=\mathcal{F}_{d}^{-1} v \mathcal{F}_{d}
$$

where $v$ on the right hand side denotes the multiplication operator by the function $v$ acting in $L^{2}\left(\mathbb{R}^{d *}\right)$. By a well known fact on multiplication operators and the unitarity of $\mathcal{F}_{d}, v(-i \nabla)$ is self-adjoint and injective. Moreover,

$$
C_{d} v(-i \nabla) \subset v(-i \nabla) C_{d}
$$

Hence $v(-i \nabla)$ is reduced by $L_{\mathbb{R}}^{2}\left(\mathbb{R}^{d}\right)$.

Let $\mathcal{D}_{d}$ be a dense subspace in $L_{\mathbb{R}}^{2}\left(\mathbb{R}^{d}\right)$ satisfying the following conditions:

(i) $\mathcal{D}_{d} \subset D(v(-i \nabla)) \cap D\left(v(-i \nabla)^{-1}\right)$.

(ii) $v(-i \nabla) \mathcal{D}_{d}$ and $v(-i \nabla)^{-1} \mathcal{D}_{d}$ are dense in $L_{\mathbb{R}}^{2}\left(\mathbb{R}^{d}\right)$.

We introduce operators $\phi_{v}(f)$ and $\pi_{v}(f)\left(f \in \mathcal{D}_{d}\right)$ as follows:

$$
\phi_{v}(f):=\Phi\left(v(-i D)^{-1} f\right), \quad \pi_{v}(f):=\Pi(v(-i D) f), \quad f \in \mathcal{D}_{d}
$$

By applying Lemma 4.3 with $\mathcal{H}=L^{2}\left(\mathbb{R}^{d}\right)$ and $T=v(-i \nabla)$, we obtain the following lemma:

Lemma $6.7\left(\mathcal{F}_{\mathrm{b}}\left(L^{2}\left(\mathbb{R}^{d}\right)\right),\left\{e^{i \phi_{v}(f)}, e^{i \pi_{v}(f)} \mid f \in \mathcal{D}_{d}\right\}\right)$ is an irreducible Weyl representation of the CCR over $\mathcal{D}_{d}$.

Theorem 6.8 Suppose that, for all $\lambda \geq 0$, the set $\left\{k \in \mathbb{R}^{d *} \mid v(k)^{2}=\lambda\right\}$ is a null set with respect to the d-dimensional Lebesgue measure. Then $\left(\mathcal{F}_{\mathrm{b}}\left(L^{2}\left(\mathbb{R}^{d}\right)\right),\left\{e^{i \phi_{v}(f)}, e^{i \pi_{v}(f)} \mid f \in\right.\right.$ $\left.\left.\mathcal{D}_{d}\right\}\right)$ has no vacuum. 
Proof. By the assumption, $\sigma_{\mathrm{p}}\left(v(-i \nabla)^{2}\right)=\sigma_{\mathrm{p}}\left(v^{2}\right)=\emptyset$. Hence, by Corollary 4.9, the desired result follows.

Two Borel measurable functions $v_{1}$ and $v_{2}$ on $\mathbb{R}^{d *}$ are said to be equal and written $v_{1}=v_{2}$ if $v_{1}(k)=v_{2}(k)$ for a.e. $k \in \mathbb{R}^{d *}$.

Lemma 6.9 Let $v_{1}$ and $v_{2}$ be functions on $\mathbb{R}^{d *}$ having the same properties as those of $v$ described above. Suppose that $v_{1} / v_{2}$ and $v_{2} / v_{1}$ are essentially bounded. Then

$$
W:=\overline{\left(v_{2}(-i \nabla)^{-1} v_{1}(-i \nabla)-v_{2}(-i \nabla) v_{1}(-i \nabla)^{-1}\right)}
$$

is bounded. Moreover, $W$ is Hilbert-Schmidt if and only if $v_{1}=v_{2}$.

Proof. Let $\hat{W}:=\mathcal{F}_{d} W \mathcal{F}_{d}^{-1}$. Then $\hat{W}=\left(v_{1}^{2}-v_{2}^{2}\right) / v_{1} v_{2}$, which is bounded. Hence $W$ is bounded.

Suppose that $W$ is Hilbert-Schmidt. Then so is $\hat{W}$. If $v_{1} \neq v_{2}$, then $\hat{W} \neq 0$. But a non-zero multiplication operator on $L^{2}\left(\mathbb{R}^{d *}\right)$ can not be Hilbert-Schmidt. Hence $v_{1}=v_{2}$.

Conversely suppose that $v_{1}=v_{2}$. Then $\hat{W}=0$. Hence $W=0$. Therefore $W$ is Hilbert-Schmidt.

Theorem 6.10 Let $v_{1}$ and $v_{2}$ be functions having the same properties as those of $v$ described above. Suppose that $v_{1} / v_{2}$ and $v_{2} / v_{1}$ are essentially bounded. Then $\left(\mathcal{F}_{\mathrm{b}}\left(L^{2}\left(\mathbb{R}^{d}\right)\right)\right.$, $\left.\left\{e^{i \phi_{v_{1}}(f)}, e^{i \pi_{v_{1}}(f)} \mid f \in \mathcal{D}_{d}\right\}\right)$ and $\left(\mathcal{F}_{\mathrm{b}}\left(L^{2}\left(\mathbb{R}^{d}\right)\right),\left\{e^{i \phi_{v_{2}}(f)}, e^{i \pi_{v_{2}}(f)} \mid f \in \mathcal{D}_{d}\right\}\right)$ are inequivalent if and only if $v_{1} \neq v_{2}$.

Proof. By the essential boundedness of $v_{1} / v_{2}$ and $v_{2} / v_{1}$, one can see that conditions (a) and (b) in Theorem 5.1 are satisfied with $T_{1}=v_{1}(-i \nabla)$ and $T_{2}=v_{2}(-i \nabla)$. Then, combining Theorem 5.1 and Lemma 6.9, we obtain the desired result.

In the case where $v_{1} / v_{2}$ and $v_{2} / v_{1}$ are not necessarily essentially bounded, we have the following theorem.

Theorem 6.11 Let $v_{1}$ and $v_{2}$ be functions having the same properties as those of $v$ described above and $v_{1} \neq v_{2}$. Let

$$
\mathcal{D}_{d, v_{1}}:=\left(v_{1}(-i \nabla) \mathcal{D}_{d}\right) \cap\left(v_{1}(-i \nabla)^{-1} \mathcal{D}_{d}\right)
$$

and

$$
T_{d, \pm}:=v_{2}(-i \nabla)^{-1} v_{1}(-i \nabla) \pm v_{2}(-i \nabla) v_{1}(-i \nabla)^{-1}
$$

Suppose that the following (a) and (b) hold:

(a) $\mathcal{D}_{d, v_{1}}$ is dense in $L_{\mathbb{R}}^{2}\left(\mathbb{R}^{d}\right)$.

(b) $T_{d,+} \mathcal{D}_{d, v_{1}}$ is dense in $L_{\mathbb{R}}^{2}\left(\mathbb{R}^{d}\right)$.

Then $\left(\mathcal{F}_{\mathrm{b}}\left(L^{2}\left(\mathbb{R}^{d}\right)\right),\left\{e^{i \phi_{v_{1}}(f)}, e^{i \pi_{v_{1}}(f)} \mid f \in \mathcal{D}_{d}\right\}\right)$ is inequivalent to $\left(\mathcal{F}_{\mathrm{b}}\left(L^{2}\left(\mathbb{R}^{d}\right)\right),\left\{e^{i \phi_{v_{2}}(f)}\right.\right.$, $\left.\left.e^{i \pi_{v_{2}}(f)} \mid f \in \mathcal{D}_{d}\right\}\right)$ 
Proof. We apply Theorem 5.6 with the following realization:

$$
\mathcal{H}=L^{2}\left(\mathbb{R}^{d}\right), T_{1}=v_{1}(-i \nabla), T_{2}=v_{2}(-i \nabla), \mathcal{D}=\mathcal{D}_{d}
$$

We need only to prove that

$$
L:=T_{d,-} T_{d,+}^{-1}
$$

is bounded and $\bar{L}$ is not Hilbert-Schmidt. It is easy to see that $L$ is bounded and

$$
\mathcal{F}_{d} \bar{L} \mathcal{F}_{d}^{-1}=\frac{1-\left(v_{2} / v_{1}\right)^{2}}{1+\left(v_{2} / v_{1}\right)^{2}} .
$$

where the right hand side is the multiplication operator by the same function. Since $v_{1} \neq v_{2}$, it follows that the operator on the right hand side is not Hilbert-Schmidt. Hence $\bar{L}$ is not Hilbert-Schmidt.

\subsection{Quantum fields in a bounded region}

It may be interesting to compare quantum fields on $\mathbb{R}^{d}$ with those on a bounded region in view of representation of CCR. Let $M$ be a bounded connected open set in $\mathbb{R}^{d}$ and consider a scalar quantum field on $M$. A Hilbert space for such a quantum field can be taken to be the boson Fock space $\mathcal{F}_{\mathrm{b}}\left(L^{2}(M)\right)$ over $L^{2}(M)$. As is well known, the Laplacian $\Delta_{0}:=\sum_{j=1}^{d} \partial^{2} / \partial x_{j}^{2}$ with domain $C_{0}^{\infty}(M)$ (the set of $C^{\infty}$-functions on $M$ with support in $M)$ is not essentially self-adjoint. Hence, to determine the one-particle dynamics in $M$, we have to fix a self-adjoint extension of $\Delta_{0}$.

Let $\Delta^{M}$ be any self-adjoint extension of $\Delta_{0}$ such that

(i) $\Delta^{M} \leq 0$;

(ii) $C^{M} \Delta^{M} \subset \Delta^{M} C^{M}$, where $C^{M}$ is the complex conjugation on $L^{2}(M)$;

(iii) The spectrum of $-\Delta_{M}$ is purely discrete. The eigenvalues of $-\Delta_{M}$ are labeled as $\left\{\lambda_{\mathbf{n}}\right\}_{\mathbf{n} \in \Gamma}$ with $\Gamma=\mathbb{N}^{d}$ or $(\{0\} \cup \mathbb{N})^{d}$, counting multiplicities, and, for some constants $c_{1}, c_{2}>0$ with $c_{1}<c_{2}$,

$$
c_{1}|\mathbf{n}|^{2} \leq \lambda_{\mathbf{n}} \leq c_{2}|\mathbf{n}|^{2}, \quad \mathbf{n} \in \Gamma
$$

Examples of $\Delta^{M}$ are the Dirichlet Laplacian $\Delta_{\mathrm{D}}^{M}$ and the Neumann Laplacian $\Delta_{\mathrm{N}}^{M}$ with a suitable additional condition on the boundary of $M$ (see, e.g., [3], [8, p.263] and $[9, \S 10.6])$. In the case where $M$ is a rectangle $\left(-L_{1} / 2, L_{1} / 2\right) \times \cdots \times\left(-L_{d} / 2, L_{d} / 2\right)$ with $L_{j}>0, j=1, \ldots, d$, one can also take $\Delta^{M}$ to be the Laplacian $\Delta_{\mathrm{P}}^{M}$ with the periodic boundary condition.

The one-particle Hamiltonian with mass $m>0$ in the present context is given by

$$
h_{m}^{M}:=\left(-\Delta^{M}+m^{2}\right)^{1 / 2}
$$

acting in $L^{2}(M)$. It follows that $h_{m}^{M}$ is a strictly positive self-adjoint operator with $h_{m}^{M} \geq$ $m>0$ and, for all $\alpha>0,\left(h_{m}^{M}\right)^{-\alpha} \in \mathfrak{B}\left(L^{2}(M)\right)$. 
By properties (ii) and (iii), there exists a CONS $\left\{f_{\mathbf{n}}\right\}_{\mathbf{n} \in \Gamma}$ of $L^{2}(M)$ such that

$$
-\Delta^{M} f_{\mathbf{n}}=\lambda_{\mathbf{n}} f_{\mathbf{n}}, \quad \mathbf{n} \in \Gamma,
$$

and each $f_{\mathbf{n}}$ is a real-valued function. Hence the subspace

$$
\mathcal{V}_{M}:=\operatorname{span}_{\mathbb{R}}\left\{f_{\mathbf{n}} \mid \mathbf{n} \in \Gamma\right\}
$$

algebraically spanned by $\left\{f_{\mathbf{n}} \mid \mathbf{n} \in \Gamma\right\}$ with real coefficients is dense in the real Hilbert space $L_{\mathbb{R}}^{2}(M)$. It follows that, for all $\alpha>0$,

$$
\mathcal{V}^{M} \subset D\left(\left(h_{m}^{M}\right)^{\alpha}\right), \quad\left(h_{m}^{M}\right)^{ \pm \alpha} \mathcal{V}^{M}=\mathcal{V}^{M} .
$$

Hence conditions (T.1) and (T.2) with $T=\left(h_{m}^{M}\right)^{1 / 2}$ in Section 4 are satisfied.

Let $\Phi^{M}(\cdot)$ be the Segal field operator on $\mathcal{F}_{\mathrm{b}}\left(L^{2}(M)\right)$ and

$$
\phi_{m}^{M}(f):=\Phi^{M}\left(\left(h_{m}^{M}\right)^{-1 / 2} f\right), \quad \pi_{m}^{M}(f):=\Phi^{M}\left(i\left(h_{m}^{M}\right)^{1 / 2} f\right), \quad f \in \mathcal{V}^{M} .
$$

Then, by Lemma 4.3,

$$
\rho_{m}^{M}:=\left(\mathcal{F}_{\mathrm{b}}\left(L^{2}(M)\right),\left\{e^{i \phi_{m}^{M}(f)}, e^{i \pi_{m}^{M}(f)} \mid f \in \mathcal{V}^{M}\right\}\right)
$$

is an irreducible Weyl representation of the CCR over $\mathcal{V}^{M}$. As for this representation, the following theorem holds:

Theorem 6.12 Let $m_{1}, m_{2}>0$ and $m_{1} \neq m_{2}$. Then $\rho_{m_{1}}^{M}$ and $\rho_{m_{2}}^{M}$ are equivalent if and only if $d \leq 3$.

Proof. We set $h_{j}:=h_{m_{j}}^{M}, j=1,2$. It is easy to check that conditions (a) and (b) in Theorem 5.1 with $T_{j}(j=1,2)$ replaced by $h_{j}$ and $\mathcal{V}=\mathcal{V}^{M}$ are satisfied. The spectrum of $\overline{h_{2}^{-1 / 2} h_{1}^{1 / 2}-h_{2}^{1 / 2} h_{1}^{-1 / 2}}$ is purely discrete and the eigenvalues are given by

$$
\mu_{\mathbf{n}}:=\frac{m_{1}^{2}-m_{2}^{2}}{\left(\lambda_{\mathbf{n}}+m_{1}^{2}\right)^{1 / 4}\left(\lambda_{\mathbf{n}}+m_{2}^{2}\right)^{1 / 4}\left[\left(\lambda_{\mathbf{n}}+m_{1}^{2}\right)^{1 / 2}+\left(\lambda_{\mathbf{n}}+m_{2}^{2}\right)^{1 / 2}\right]}, \quad \mathbf{n} \in \Gamma .
$$

By (6.3), we have

$$
\frac{\left|m_{1}^{2}-m_{2}^{2}\right|}{2 c} \frac{1}{|\mathbf{n}|^{2}+\mathbf{1}} \leq\left|\mu_{\mathbf{n}}\right| \leq \frac{\left|m_{1}^{2}-m_{2}^{2}\right|}{2 c_{1}} \frac{1}{|\mathbf{n}|^{2}}, \quad \mathbf{n} \in \Gamma \backslash\{\mathbf{0}\},
$$

where $c:=\max \left\{c_{2}, m_{1}^{2}, m_{2}^{2}\right\} . \sum_{\mathbf{n} \in \Gamma} \mu_{\mathbf{n}}^{2}$ converges if and only if $d \leq 3$. Hence $\overline{h_{2}^{-1} h_{1}-h_{2} h_{1}^{-1}}$ is Hilbert-Schmidt if and only if $d \leq 3$. Thus an application of Theorem 5.1 yields the desired result.

Remark 6.13 Theorem 6.12 suggests that, in the case $d=1,2,3$, the inequivalence of $\rho_{m}$ 's for different masses as in Theorem 6.4 comes from "infinity in space".

Finally we consider the case where $m_{1}>0$ and $m_{2}=0$. In this case, if $0 \notin \sigma\left(\Delta^{M}\right)$ (e.g. the case $\Delta^{M}=\Delta_{\mathrm{D}}^{M}$ ), then the proof of Theorem 6.12 works without essential change. Hence we have the following result:

Theorem 6.14 Let $m>0$ and $0 \notin \sigma\left(\Delta^{M}\right)$. Then $\rho_{m}^{M}$ is equivalent to $\rho_{0}^{M}$ if and only if $d \leq 3$. 


\section{Acknowledgement}

This work is supported by the Grant-In-Aid No.15K04888 for Scientific Research from Japan Society for the Promotion of Science (JSPS).

\section{References}

[1] A. Arai, Fock Spaces and Quantum Fields (in Japanese), Nippon-hyoronsha, Tokyo, 2000.

[2] J. Dereziński and C. Gérard, Mathematics of Quantization and Quantum Fields, Cambridge University Press, Cambridge, 2013.

[3] T. Ikebe, Eigenvalue Problem in Mathematical Physics: Discrete Spectrum (in Japanese), Sangyou-Tosho, Tokyo, 1976.

[4] C. R. Putnam, Commutation Properties of Hilbert Space Operators and Related Topics, Springer, Berlin, Heidelberg, 1967.

[5] M. Reed and B. Simon, Methods of Modern Mathematical Physics I: Functional Analysis, Academic Press, New York, 1972.

[6] M. Reed and B. Simon, Methods of Modern Mathematical Physics II: Fourier Analysis, Self-Adjointness, Academic Press, New York, 1975.

[7] M. Reed and B. Simon, Methods of Modern Mathematical Physics III: Scattering Theory, Academic Press, New York, 1979.

[8] M. Reed and B. Simon, Methods of Modern Mathematical Physics IV: Analysis of Operators, Academic Press, New York, 1978.

[9] K. Schmüdgen, Unbounded Self-adjoint Operators on Hilbert Space, Springer, Dordrecht, 2012.

[10] D. Shale, Linear symmetries of free boson fields, Trans. Amer. Math. Soc. 103 (1962), 149-167. 